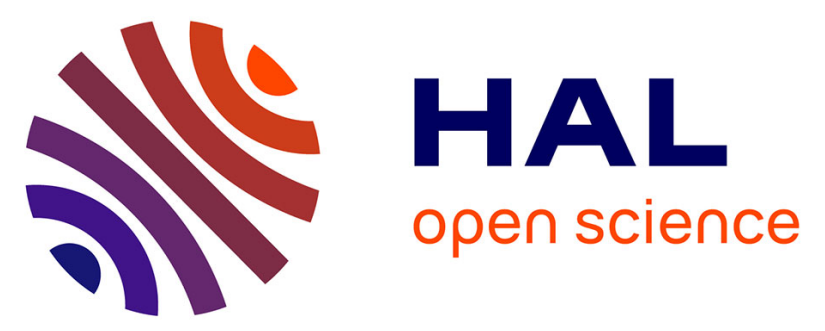

\title{
Granule cells in the infrapyramidal blade of the dentate gyrus are activated during paradoxical (REM) sleep hypersomnia but not during wakefulness: a study using TRAP mice
}

\author{
Risa Yamazaki, Dianru Wang, Anna de Laet, Renato Maciel, Claudio \\ Agnorelli, Sébastien Cabrera, Sébastien Arthaud, Paul-Antoine Libourel, \\ Patrice Fort, Hyunsook Lee, et al.
}

\section{- To cite this version:}

Risa Yamazaki, Dianru Wang, Anna de Laet, Renato Maciel, Claudio Agnorelli, et al.. Granule cells in the infrapyramidal blade of the dentate gyrus are activated during paradoxical (REM) sleep hypersomnia but not during wakefulness: a study using TRAP mice. SLEEP, 2021, 44 (12), pp.zsab173. 10.1093/sleep/zsab173 . hal-03400920

\author{
HAL Id: hal-03400920 \\ https://hal.science/hal-03400920
}

Submitted on 9 Nov 2021

HAL is a multi-disciplinary open access archive for the deposit and dissemination of scientific research documents, whether they are published or not. The documents may come from teaching and research institutions in France or abroad, or from public or private research centers.
L'archive ouverte pluridisciplinaire HAL, est destinée au dépôt et à la diffusion de documents scientifiques de niveau recherche, publiés ou non, émanant des établissements d'enseignement et de recherche français ou étrangers, des laboratoires publics ou privés. 


\section{OXFORD}

\section{SLEEP}

\section{Granule cells in the infrapyramidal blade of the dentate gyrus are activated during paradoxical (REM) sleep hypersomnia but not during wakefulness: a study using TRAP mice}

\begin{tabular}{|c|c|}
\hline Journal: & Sleep \\
\hline Manuscript ID & Draft \\
\hline Manuscript Type: & Original Article \\
\hline Date Submitted by the Author: & $\mathrm{n} / \mathrm{a}$ \\
\hline Complete List of Authors: & $\begin{array}{l}\text { Yamazaki, Risa; University Claude Bernard } \\
\text { Lyon 1, UMR 5292 CNRS/U1028 INSERM } \\
\text { Wang, Dianru; Universite Claude Bernard } \\
\text { Lyon 1, UMR 5292 CNRS/U1028 INSERM } \\
\text { DeLaet, Anna; Universite Claude Bernard } \\
\text { Lyon 1, UMR 5292 CNRS/U1028 INSERM } \\
\text { Maciel, Renato; Universite Claude Bernard } \\
\text { Lyon 1, UMR 5292 CNRS/U1028 INSERM } \\
\text { Agnorelli, Claudio; Université Claude } \\
\text { Bernard Lyon 1, UMR } 5292 \text { CNRS/U1028 } \\
\text { INSERM } \\
\text { Cabrera, Sébastien; Université Claude } \\
\text { Bernard Lyon 1, UMR } 5292 \text { CNRS/U1028 } \\
\text { INSERM } \\
\text { Arthaud, Sébastien; Université Claude } \\
\text { Bernard Lyon 1, UMR 5292 CNRS/U1028 } \\
\text { INSERM } \\
\text { Libourel, Paul-Antoine; University Claude } \\
\text { Bernard Lyon 1, UMR 5292 CNRS/U1028 } \\
\text { INSERM } \\
\text { Fort, Patrice; Université Claude Bernard } \\
\text { Lyon 1, UMR 5292 CNRS/U1028 INSERM } \\
\text { Lee, Hyunsook; Université Claude Bernard } \\
\text { Lyon 1, UMR 5292 CNRS/U1028 INSERM; } \\
\text { Konkuk University, Department of } \\
\text { Anatomy; Konkuk University, Research } \\
\text { Institute of Medical Science, School of } \\
\text { Medicine } \\
\text { Luppi, Pierre-Herve; Université Claude } \\
\text { Bernard Lyon 1, UMR 5292 CNRS/U1028 } \\
\text { INSERM }\end{array}$ \\
\hline \multicolumn{2}{|l|}{ Keywords: } \\
\hline $\begin{array}{r}\text { Please select below if your paper belongs to the Call for } \\
\text { Papers. <p>SUBMISSION CRITERIA for the Call for Papers } \\
\text { can be found <a } \\
\text { href="https://academic.oup.com/sleep/pages/big_data_cfp" }\end{array}$ & \\
\hline
\end{tabular}


1

2

3

4

5

6

7

8

9

10

11

12

13

14

15

16

17

18

19

20

21

22

23

24

25

26

27

28

29

30

31

32

33

34

35

36

37

38

39

40

41

42

43

44

45

46

47

48

49

50

51

52

53

54

55

56

57

58

59

60

\begin{tabular}{|r|l|}
\hline target $=$ "_new" $>$ here $</$ a $>$ : & \\
\hline Section: & \\
\hline Keywords Pick List: & \\
\hline Other Keywords: & \\
\hline
\end{tabular}

\section{SCHOLARONE ${ }^{\mathrm{T}}$ Manuscripts}

https://mc.manuscriptcentral.com/jsleep 
Title: Granule cells in the infrapyramidal blade of the dentate gyrus are activated during paradoxical (REM) sleep hypersomnia but not during wakefulness: a study using TRAP mice

Risa Yamazaki1 ${ }^{1, *}$, Dianru Wang ${ }^{1, *}$, Anna De Laet ${ }^{1 *}$, Renato Maciel, Claudio Agnorelli, Sébastien Cabrera, Sébastien Arthaud ${ }^{1}$, Paul-Antoine Libourel ${ }^{1}$, Patrice Fort ${ }^{1}$, Hyunsook Lee ${ }^{1,2,3,+}$, Pierre-Hervé Luppi $^{1, \dagger}$

${ }^{1}$ Team "SLEEP", Centre de Recherche en Neurosciences de Lyon (CRNL), UMR 5292 CNRS/U1028 INSERM and Université de Lyon, Lyon I, Neurocampus-Michel Jouvet, 95 Boulevard Pinel, 69500 Bron, France

${ }^{2}$ Department of Anatomy and ${ }^{3}$ Research Institute of Medical Science, School of Medicine, Konkuk University, 05029 Seoul, South Korea

${ }^{*}$ Equally contributed, ${ }^{+}$Coresponsible

\section{Correspondence:}

Pierre-Hervé Luppi, PhD. luppi@sommeil.univ-lyon1.fr

Hyunsook Lee, PhD. hyunsook.lee@kku.ac.kr 


\begin{abstract}
Study Objectives

Determine whether in the hippocampus and the supramammillary nucleus (SuM) the same neurons are reactivated when mice are exposed one week apart to two periods of wakefulness (W-W), paradoxical sleep rebound (PSR-PSR) or a period of W followed by a period of PSR (W-PSR)
\end{abstract}

\title{
Methods
}

We combined the innovative TRAP2 mice method in which neurons expressing cFos permanently express tdTomato after tamoxifen injection with cFos immunohistochemistry.

\section{Results}

We found out that a large number of tdTomato + and cFos + cells are localized in the dentate gyrus (DG) after PSR and W while CA1 and CA3 contained both types of neurons only after W. The number of cFos + cells in the infrapyramidal but not the suprapyramidal blade of the DG was positively correlated with the amount of PS. In addition, we did not find double-labeled cells in the DG whatever the group of mice. In contrast, a high percentage of CA1 neurons were double-labeled in W-W mice. Finally, in the supramammillary nucleus, a large number of cells were double-labeled in W-W, PSR-PSR but not in W-PSR mice.

\section{Conclusions}

Altogether, our results are the first to show that different neurons are activated during W and PS in the supramammillary nucleus and the hippocampus. Further, we showed for the first time that granule cells of the infrapyramidal blade of the DG are activated during PS but not during W. Further experiments are now needed to determine whether these granule cells belong to memory engrams inducing memory reactivation during $\mathrm{PS}$.

Keywords: sleep, dentate gyrus, granule cells, memory, cFos 


\section{Introduction}

Paradoxical sleep (PS), a.k.a. rapid eye movement (REM) sleep, is characterized by vivid dreams, REM, muscle atonia, hippocampal theta oscillations and a desynchronized cortical EEG similar to that of waking $(\mathrm{W})^{1-3}$. Based on the expression of cFos protein as a marker for neuronal activation, we recently showed in rats that only a few cortical and limbic structures namely the dentate gyrus (DG) of the hippocampus, the claustrum, cortical amygdaloid nucleus, post-, pre- and parasubiculum, medial entorhinal and retrosplenial cortices are activated during a PS rebound (PSR) occurring after 72h of PS deprivation (PSD) using the classical flowerpot method ${ }^{4}$. In contrast, all cortical structures are activated during $\mathrm{W}$ induced by exposing the rats to an open field for $3 \mathrm{~h}$. Further, we also showed that the DG was the only cortical structure containing significantly more $\mathrm{cFos}^{+}$neurons during PSR than during W. In addition, we showed that the dorsal CA1 and CA3 contained a large number of $\mathrm{cFos}^{+}$neurons during $\mathrm{W}$ but that there were nearly devoid of neurons during PSR ${ }^{4}$. We finally showed that the lateral supramammillary nucleus (SuML) was the only subcortical structures containing neurons activated during PSR and projecting to the DG. Further, lesion of the SuML induced a disappearance of cFos staining in the dorsal but not the ventral $\mathrm{DG}^{4,5}$.

Although our results showed that the DG, but not CA1 and CA3, are more activated during PSR than $\mathrm{W}$, we could not rule out the hypothesis that such activation was due to the semi-chronic stress induced by the flowerpot method used for PSD. Further, conditional cFos method did not allow us to examine whether the same neurons are reactivated across several PS episodes nor whether neurons activated during W could be reactivated during subsequent PS episodes. To resolve the specificity issue and determine whether reactivation of the same neurons occurs, we used the new 'targeted recombination in active populations (TRAP)' method that allows to permanently tag neurons activated by defined stimuli 6-8. We already validated this method in our recent study on the activation of lateral hypothalamic neurons during PSR and $\mathrm{W}^{9}$. The transgenic TRAP2 mice express tamoxifen-dependent CreER ${ }^{\mathrm{T} 2}$ recombinase under $\mathrm{cFos}$ promoter. $\mathrm{CreER}^{\mathrm{T} 2}$ can only undergo recombination when 4-hydroxytamoxifen (4-OHT, an active metabolite of tamoxifen) is present with a time window of approximately $4 \mathrm{~h}$ centered 


\section{Materials and Methods}

\section{Animals}

All experiments were conducted in accordance with the French and European Community guidelines for using animals in research and were approved by the institutional animal care and committee of the University of Lyon 1 and NEUROCAMPUS (Project APAFIS\#21351). Both male and female of double heterozygous Fos ${ }^{2 \mathrm{~A}-\mathrm{iCreER} ; \mathrm{R} 26^{\mathrm{Ai} 14}}$ (TRAP2) mice, generated by crossing Fos ${ }^{2 \mathrm{~A}-\mathrm{iC} \text { reER/+}}$ (TRAP2) mice to R26 AI14/+ (AI14) mice7. TRAP2 mice were kindly gifted by Dr. Liqun Luo from Stanford University. In all experiments, age 8-12 weeks old mice were prepared. Transgenic mice were housed individually and placed under a constant light/dark cycle (light on from 8:00 a.m. to 8:00 p.m.).

\section{Experimental design}

Animals were divided into 3 groups as illustrated in Fig. 1B: Waking (W)-W group (n=3), paradoxical sleep rebound (PSR)-PSR group $(n=3)$, and a W-PSR group $(n=5)$. The $W-W$ group was subjected to 
two periods of $\mathrm{W}$ separated by one week. The PSR-PSR group was subjected to a deprivation and rebound of PS one week apart. Finally, the W-PSR group was subjected to an induction of W followed by a PS deprivation and rebound one week later. W and PSR protocols are described below.

\section{Surgery}

All animals in the PSR-PSR and W-PSR groups were anesthetized with Ketamine and Xylazine (100/10 $\mathrm{mg} / \mathrm{kg}$. i.p.). Then, the top of the head was shaved and the mice were placed in a stereotactic frame with a heating pad underneath. Two stainless screws were fixed in the parietal part (AP: -2.0, ML: 1.5 from bregma) and one in the frontal part (AP: +2.0, ML: 1.0 from bregma) of the skull, whereas the reference electrode for unipolar EEG recoding was fixed in the occipital part (AP: -5.0, ML: 0.0 from bregma). Two wire electrodes were inserted into the neck muscles for bipolar EMG recordings. All leads were connected to a miniature plug (Plastics One, Roanoke, VA) that was cemented on the skull.

\section{Polysomnography and analysis of vigilance states}

Animals in the PSR-PSR and W-PSR groups were allowed to recover from surgery for 1 week in their home cage before being habituated to the recording conditions for 3 days. They were connected to a cable attached to a slip-ring commutator to allow free movement within the barrel. Unipolar EEG and bipolar EMG signals were amplified (MCP-PLUS, Alpha-Omega Engineering, Israel), digitized at 512 Hz, and transferred to Slip Analysis v 2.9.8 software (View Point, Civrieux, France). The analysis was done as previously described ${ }^{4}$ with slight modifications. Briefly, the vigilance states of $5 \mathrm{~s}$ episodes of EEG and EMG recordings were identified by a visual check of polygraphic signals according to the criteria described in detail elsewhere (Example of representative EEG/EMG signals from each state are shown in Fig. 1C). Wakefulness, SWS and PS were quantified during the last 2 hours (10am to 12am) before euthanasia and in all PSR animals. The amount of the three states between 10am-12am of baseline recordings ( 3 days before PSR, thus 1 day before PSD) from the same animals were quantified as well as a control. 
4-hydroxytamoxifen (4-OHT) was prepared as described previously ${ }^{8}$. Briefly, 4-OHT (Cat\# H6278 Sigma Aldrich, St. Luis, MO) was dissolved at $20 \mathrm{mg} / \mathrm{mL}$ in absolute ethanol by ultrasonic water bath at $37^{\circ} \mathrm{C}$ for $10 \mathrm{~min}$, and was then aliquoted and stored at $-20^{\circ} \mathrm{C}$ as a stock solution. Before using, corn oil (Sigma Aldrich) was added to the thawed stock solution to replace the ethanol and to obtain 10 $\mathrm{mg} / \mathrm{mL} 4-\mathrm{OHT}$, and then the ethanol was evaporated at $37^{\circ} \mathrm{C}$. The $10 \mathrm{mg} / \mathrm{mL} 4-\mathrm{OHT}$ solutions were used the same day or one day after preparation. All animals were injected intraperitoneally (i.p.) with 50 $\mathrm{mg} / \mathrm{kg}$ 4-OHT after $2 \mathrm{~h}$ from the beginning of either Wakefulness or PSR paradigm.

\section{Wakefulness}

The waking protocol was used to induce a continuous period of wakefulness during 4 hours. To maintain the animals awake, two mice were placed into a square $45^{*} 45 \mathrm{~cm}$ white open field box together with wood tips and small objects. Food and water were freely available in the box. During $4 \mathrm{~h}$, the animals were permanently monitored by the web-camera from a different room to check whether the animals were awake. The animals were gently touched by a soft tissue when they became inactive/drowsy.

\section{Paradoxical sleep deprivation and rebound (PSD and PSR)}

The PSD was performed like in our previous report ${ }^{9,11}$. Briefly, the mouse was placed in a transparent individual barrel with a movable floor connected to a small piston controlled by an electromagnet. All the deprivations started at 10:00 am (ZT 2). During the recording, as soon as PS appeared and was detected on-line by our algorithm, a TTL electrical signal was sent to a current generator controlling the instantaneous onset of the electromagnet. This caused a few slight movements (up/down) of the floor that woke up the animal after only 5 seconds of the detection of PS10,11. After $48 \mathrm{~h}$ of PSD, stimulation 
automatically stops at 10:00 am without human intervention. Then animals were allowed to recover PS in the same barrel.

\section{Perfusion}

All animals were deeply anesthetized with pentobarbital (400 mg/kg, i.p.) and were perfused heparinadded Ringer's lactate solution followed by $4 \%$ paraformaldehyde/PBS ( $\mathrm{pH}$ 7.4) for pre-fixation. The brains were post-fixed with $4 \%$ paraformaldehyde for one night at $4^{\circ} \mathrm{C}$. The brains were then stored in $30 \%$ sucrose/PB for two days at $4^{\circ} \mathrm{C}$.

\section{Immunohistochemistry}

Brains were frozen with methylbutane placed on a dry ice at around $-30^{\circ} \mathrm{C}$. Then the brain was sliced in $30 \mu \mathrm{m}$ thick coronal sections, and stored at $-20^{\circ} \mathrm{C}$ in cryoprotective solution containing $20 \%$ glycerol and $30 \%$ Ethylene glycol in 0.05 M PB (pH 7.4). They were successively incubated in (i) rabbit anticFos antibody (1:15000, \#ABE457, Millipore, Burlington, MA) in PBST for 2 days at $4{ }^{\circ} \mathrm{C}$; (ii) a biotinylated anti-rabbit immunoglobulin G (IgG) solution (\#BA-9500, Vector Laboratories, Burlingame, CA); and (iii) an ABC-horseradish peroxidase solution (Elite kit \#PK-6100, Vector Laboratories) for 90 min at room temperature. Finally, the sections were immersed in DAB solution $(0.025 \% 3,3$ 'diaminobenzidine-4 $\mathrm{HCl}$ (DAB; Sigma Aldrich) and 0.003\% hydrogen peroxide in $0.05 \mathrm{M}$ Tris-HCl buffer ( $\mathrm{pH} 7.6)$ ) with $0.6 \%$ nickel ammonium sulfate to obtain a black reaction product. After washing for 10 min with PBST, the cFos-stained sections were reincubated in the rat anti-mCherry antibody (1:100000, \#M11217, Invitrogen, Carlsbad, CA), biotinylated anti-rat antibody, and an ABChorseradish peroxidase solution. Then the sections were put in the DAB solution to obtain a brown reaction product. After mounting the sections on slide glass, the slides were sequentially submerged in $70 \%, 90 \%$ and $100 \%$ ethanol for 2 min each. Lastly, the slide was submerged in toluene for 2 min preceded by an immersion in a xylene and alcohol substitute (Ottix ${ }^{\circledR}$, DiaPath, Martinengo, Italy) for the same amount of time. Finally, the slides were cover-slipped with a slide mounting medium (DPX 
Mountant, Sigma Aldrich). The data acquisition was done by using Morphostrider software (Extra Nova, La Rochelle, France) with Axioskop microscope (Carl Zeiss, Oberkochen, Germany).

\section{Immunofluorescence}

Sections were incubated with the same rabbit anti-cFos antibody $(1: 250)$ that for DAB staining in PBST for $24-48 \mathrm{~h}$ at $4{ }^{\circ} \mathrm{C}$. Following washes, they were incubated in AlexaFluor 488-conjugated secondary antibody (Jackson Immunoresearch Lab., West Grove, PA) in PBST for $2 \mathrm{~h}$ at room temperature. Following washes with phosphate buffer (PB), sections were mounted, dried, and cover-slipped with prolong Gold anti-fade reagent containing 4',6-diamidino-2-phenylindole (DAPI) (Molecular Probes, Eugene, OR) and stored at $4{ }^{\circ} \mathrm{C}$. For image processing, we utilized ZEN 2010 software with confocal laser scanning microscope (LSM 800, Carl Zeiss) equipped with blue argon (488 nm), green helium neon $(543 \mathrm{~nm})$, and red helium neon $(633 \mathrm{~nm})$ lasers. For image acquisition, 'best signal' was selected in SMART-setup tool. Z-stack procedure with an interval of $1.0 \mu \mathrm{m}$ was employed and processed with ZEN software to stack the images.

\section{Cell counting}

The atlas of George Paxinos and the Allen Brain Reference Atlases (Adult Mouse) were used as references for all structures. For the DAB-stained sections, drawings of structures with plotting of $\mathrm{cFos}^{+}$ and/or tdTomato ${ }^{+}$neurons each group were made with an Axioskop microscope (Carl Zeiss) equipped with a motorized XY-sensitive stage and a video camera connected to a computerized image analysis system, Mercator software (Explora Nova, La Rochelle, France) and Morphostrider software (Explora Nova). The $\mathrm{cFos}^{+}$, tdTomato ${ }^{+}$, and double $\left(\mathrm{cFos}^{+} / \mathrm{tdTomato}^{+}\right)$neurons were plotted and counted manually with the Mercator software in animals on hemi-sections taken at the same level. In case of the SuM, since the structure cannot be separated in left and right parts, number of neurons counted in the entire structure (one SuMM, and bilateral SuMLs) was divided by 2. We used DAB/DAB-nickel doublestained section for counting neurons and drawing structures. Confocal imaging was used for illustration 
purpose and to confirm that our identification of double-stained neurons using DAB and DAB-nickel was accurate. For the immunofluorescent sections, double-labeled neurons were confirmed for genuine labeling using a single channel illumination. The counting tool in the Adobe Photoshop CS6 (64 Bit) was used; cells were considered labeled when they exhibited clear cytoplasmic (i.e., tdTomato) or nuclear (i.e., cFos) morphology. For quantitative analysis, at least three animals per group (W-W; n=3, PSR-PSR; $n=3$, W-PSR; $n=5$ ) were selected.

\section{Statistical analysis}

Wilcoxon Signed-Ranks Test was performed on the amount of all states in control and in PSR condition (Fig. 1D-G, $\mathrm{n}=8$, sleep data for control and PSR conditions was obtained for each mice at the same time of the day) and on the number of labeled neurons for the same animals (Fig. 2A and B; SuMM vs SuML, Fig. 5C and D; infrapyramidal vs suprapyramidal, or core vs shell). Mann-Whitney's U test was chosen for the analysis of the number of $\mathrm{cFos}^{+}$or tdTomato ${ }^{+}$neurons between two independent groups (Figs. 2A and B, 3G and O, 4C and D; W vs PSR). Kruskal-Wallis followed by a Dunn's multiple comparison test was performed on the number of labeled neurons and $\%$ of reactivation for each structure across experimental groups (Figs. 2F, 3H, and 4H). Statistical significance of the correlation between the amount of PS and the number of cFos was calculated by Pearson's analysis (Fig. 5E).

\section{Results}

\subsection{Sleep analysis and controls}

We divided the animals into 3 groups as illustrated in Fig. 1B: a Waking (W)-W group, paradoxical sleep rebound (PSR)-PSR group, and a W-PSR group. To obtained a PSR, the automated PS deprivation (PSD) system developed in our team was used ${ }^{9-11}$ (see also Materials and Methods). Like when using the flowerpot method in mice ${ }^{12}$ and the automated system in rats ${ }^{10}$, all animals showed during PSR a significantly higher amount of PS and lower amount of W without modification of SWS compared with 
control (Wilcoxon signed rank test, $\mathrm{n}=8, p=0.0078,0.23$, and 0.0039 for $\mathrm{W}, \mathrm{SWS}$, and PS, respectively) (Fig. 1D, Fand G). The amount of PS peaked during the first $30 \mathrm{~min}$ of PSR (34\% of total time), and then progressively decreased but stayed significantly higher than control at least throughout the first 2 $\mathrm{h}$ of PSR (Wilcoxon signed rank test, $\mathrm{n}=8, p=0.0039,0.0039,0.00039$, and 0.020 for each half-hour time point) (Fig. 1E).

We then verified that in all structures examined (i.e. SLD, SuM, and HIP), the number of $\mathrm{cFos}^{+}$neurons was statistically identical in the PSR-PSR and the W-PSR groups, as well as that of tdTomato ${ }^{+}$neurons in the W-W group and the W-PSR group (Table 1) indicating the high reproducibility of the cFos and tdTomato staining when obtained for PSR or W in two different groups of mice. Therefore, for all our analyses (Figs. 2A and B, 3G, 4G, and 5C and D), the number of $\mathrm{cFos}^{+}$neurons during PSR was calculated by merging the PSR-PSR and W-PSR groups and that of tdTomato ${ }^{+}$neurons during W by merging the W-W and the W-PSR groups.

As a control, we first counted the number of neurons in the sublaterodorsal tegmental nucleus (SLD) which contains the neurons responsible for the onset and maintenance of $\mathrm{PS}^{13}$. In the PSR-PSR group, the SLD contained a significant number of tdTomato and cFos labeled neurons ( $\mathrm{n}=3,24.0$ (IQR=1.0) and 20.0 (IQR=2.0), respectively). Moreover, 26.1\% $(\mathrm{IQR}=4.6)$ of the tdTomato ${ }^{+}$neurons were double labeled (expressing cFos) ( $\mathrm{n}=3$, Fig. 1H and Table 1). In contrast, in the W-PSR group, the SLD contained less tdTomato ${ }^{+}$neurons than after PSR $(n=2,14.0(\mathrm{IQR}=3.0))$ and only $10.4 \%(\mathrm{IQR}=1.3)$ of them were double-labeled.

\subsection{Supramammillary nucleus}

The supramammillary nucleus (SuM) can be divided into a lateral and a medial part (SuML and SuMM, respectively $)^{4}$. We first examined the ratio of the number of $\mathrm{cFos}^{+}$and tdTomato ${ }^{+}$neurons for the same condition to examine the efficiency of the TRAP method. In the W-W group, the ratios of tdTomato ${ }^{+}$ versus $\mathrm{cFos}^{+}$neurons in the SuMM and SuML were of $0.4(\mathrm{IQR}=0.1)$ and $0.6(\mathrm{IQR}=0.1)$, respectively. 
In the PSR-PSR group, the ratios were of $0.5(\mathrm{IQR}=0.3)$ in the SuMM and $0.3(\mathrm{IQR}=0.2)$ in the SuML. These results indicate that the TRAP method is efficient for the SuM.

In the $\mathrm{W}$ condition, the SuMM and SuML contained similar significant numbers of $\mathrm{cFos}^{+}$neurons, 53.0 $(\mathrm{IQR}=9.5)$ and $51.0(\mathrm{IQR}=19.5)$, respectively (Fig. 2A and $\mathrm{C}$, table 1). The number of $\mathrm{cFos}^{+}$neurons was significantly higher during W than during PSR in the SuMM but not in the SuML $(p=0.0061$ and $\mathrm{p}=0.25$, respectively, Fig. 2A). During PSR, a significantly higher number of $\mathrm{cFos}^{+}$neurons was localized in the SuML than in the SuMM (SuML, 45.8 (IQR=29.9); SuMM, 18.3 (IQR=4.5), $p=0.0039)$ (Fig. 2A and D).

The SuMM and the SuML contained a similar number of tdTomato ${ }^{+}$neurons during $\mathrm{W}$ and PSR (SuMM: W, 18.5 (IQR=10.88), PSR, 10 (IQR=5.25); $p=0.085$ ) (SuML: W, 31.75 (IQR=18.0); PSR, 22 $(\mathrm{IQR}=8.5) ; p=0.33)$. A higher number of tdTomato ${ }^{+}$neurons was localized in the SuML than in the SuMM in PSR but it did reach statistical significance (SuML, 22.0 (IQR=8.5); SuMM, 10.0, (IQR=5.3)); $p=0.13)($ Fig. 2B)

Finally, the percentage of double-labeled cells over the total number of tdTomato ${ }^{+}$cells in the W-W group was very high in the SuMM (70 (IQR=13.1)\%) and the SuML (50.0 (IQR=14.6) \%) (Fig. 2C and F, Table 1). The percentage of double labeled cells in the PSR-PSR group was also high in the SuML (48.4 (IQR=4.2) \%) and to a minor extent the SuMM (23.5 (IQR=17.0) \%) (Fig. 2D and F, Table 1). In the W-PSR group, the SuMM (13.8 (IQR=14.3) \%) and SuML (19.4 (IQR=10.6) \%) contained a significantly lower percentage of double-labeled neurons than in the W-W and PSR-PSR groups (WPSR vs W-W in SuMM; $p=0.008$, W-PSR vs W-W and W-PSR vs PSR-PSR in SuML; $p=0.019$ and 0.028, respectively, Fig. 2E and F, Table 1).

In agreement with our previous report ${ }^{4}$, our results show that the SuML is more activated than the SuMM during PSR whereas both structures are similarly activated during W. In addition, the high reactivation rate in the SuM both in W-W and PSR-PSR conditions indicate that the TRAP method is very efficient for that structure. Finally, we show for the first time that different SuM neurons are activated during PSR and W. 


\subsection{Hippocampus}

Since the hippocampus is an extended structure with distinct functions along the rostro-caudal axis ${ }^{14}$, we divided it into a rostral and a caudal part (distance from Bregma: -1.8 and -3.4 , respectively).

During W (W-W group), the pyramidal cell layers of rCA1 (129.0 (IQR=107.5)) and to a minor extent rCA3 (60.0 $(\mathrm{IQR}=27.0))$ and the granular cell $(\mathrm{GC})$ layer of $\mathrm{rDG}(63.0(\mathrm{IQR}=8.0))$ of the rostral hippocampus (rHIP) contained a large number of $\mathrm{cFos}^{+}$neurons (Fig. 3A, B and G, Table 1). During PSR (PSR-PSR and W-PSR groups), the GC layer of the rDG contained more cFos $^{+}$neurons (96 $(\mathrm{IQR}=30.5))$ than during $\mathrm{W}$ but it did not reach statistical significance $(p=0.14$, Fig. $2 \mathrm{C}-\mathrm{G}$, Table1). rCA1 and rCA13 contained nearly no neurons during PSR.

Like for the rHIP, a large number of $\mathrm{cFos}^{+}$neurons were localized in the caudal Hip (cHIP), during W (Fig. 4A, B, G, and Table 1). Notably, the cCA1 (530 (IQR=303.5)) contained more cFos $^{+}$ neurons than the rCA1 although it did not reach statistical significance $(p=0.125)$. There was also a similar number of cFos + neurons in the rostral and caudal CA3 $(p=0.63)$ and DG $(p=0.63)$ (Table 1). During PSR (PSR-PSR and W-PSR groups), the cDG contained a large number of $\mathrm{cFos}^{+}$cells (52.5 $(\mathrm{IQR}=49.75))$ like during $\mathrm{W}(73.0(\mathrm{IQR}=46.5))(p=0.24$, Fig. 4G). In addition, the cCA1 contained a significant number of $\mathrm{cFos}^{+}$cells during PSR in contrast to rCA1 ( $\left.\mathrm{p}=0.0039\right)$. However, it represented ten times less cells than during $\mathrm{W}(p=0.0061)$. Finallly, like rCA3, cCA3 contained significantly fewer neurons expressing cFos during PSR than $\mathrm{W}(\mathrm{p}=0.0061$, Fig. $4 \mathrm{G})$.

Both for W-W and PSR-PSR groups, the ratio of tdTomato ${ }^{+}$versus cFos + cells in the DG was around one fourth whereas in CA1 and CA3, it was of around one tenth (Table1).

In rCA1, the number of tdTomato ${ }^{+}$neurons was very low and did not significantly differ between $\mathrm{W}$ (6.3 (IQR=7.3)) and PSR (PSR-PSR, $7.0(\mathrm{IQR}=3.5) ; p=0.29)$ conditions. In contrast a significant number of tdTomato ${ }^{+}$neurons was located in cCA1 in $\mathrm{W}(67(\mathrm{IQR}=47.5))$ and to a minor extent during PSR (31 (IQR=20.5); $p=0.063)$. Further, during PSR, cCA1 contained significantly more cells than rCA1 $(p<0.0001)$. In the rDG, the number of tdTomato ${ }^{+}$neurons was slightly higher during PSR (26 
$(\mathrm{IQR}=9.5)$ than during $\mathrm{W}(15.25(\mathrm{IQR}=17.5))$ but it did not reach statistical significance $(p=0.14)$. Similarly, in the cDG, the number of tdTomato + neurons was nearly similar between conditions (W, 10.0 (IQR=15.3); PSR, 14.5 (IQR=5.5)). Finally, in cCA3 (W, $3.8(\mathrm{IQR}=4.5) ; \mathrm{PSR}, 1,(\mathrm{IQR}=0))$ and rCA3 (W, 5.0 (IQR =5.25); PSR, 4.5 (IQR=4.5)), a few tdTomato ${ }^{+}$neurons was observed during $\mathrm{W}$ and PSR.

Concerning reactivation, a high percentage of tdTomato cells were double-labeled in rCA1 and cCA1 in the $\mathrm{W}-\mathrm{W}$ group $(33.3$ (IQR=5.6) \% and $46.2(\mathrm{IQR}=9.7) \%$, respectively) whereas no doublelabeled cell was observed in the rostral and caudal CA3 and DG (Figs. 3A, B, and H, 4A, B, and H, and Table 1) despite the high number of tdTomato ${ }^{+}$cells in particular in the DG. Similarly, in the PSR-PSR group, despite the high number of $\mathrm{cFos}^{+}$and tdTomato ${ }^{+}$cells in the DG, nearly no double-labeled cells were found (Figs. 3C, D, and H, 4C, D, and H, and Table 1). Nearly no double-labeled cells were also seen in the cCA1 during PSR despite the presence of a substantial number of tdTomato ${ }^{+}$and $\mathrm{cFos}^{+}$cells. Finally, in the W-PSR group, there was almost no neurons coexpressing cFos and tdTomato in both rostral and caudal HIP including the DG although it contained a large number of $\mathrm{cFos}^{+}$and tdTomato + neurons.

\subsection{Suprapyramidal and infrapyramidal blades of the $D G$}

Among the structures investigated, the DG has several interesting features. One was the extremely low reactivation rate in all group. The second was the difference in distribution of neurons activated during $\mathrm{W}$ and PSR. Indeed, the neurons activated during $\mathrm{W}$ in the DG were localized mainly in the suprapyramidal blade and not in the infrapyramidal blade, while those activated during PSR were spread out throughout the two blades (Fig. 5B). To analyze quantitatively the distribution of the cells, we divided the GC layer into four parts as illustrated in Fig. 5A. We first marked the boundary (called also "crest") between the suprapyramidal and infrapyramidal blades in the rDG by a red line (Fig. 5A). Then we further divided each blade into shell and core parts (close to the molecular layer and the Hilus, 
respectively ${ }^{15}$ ) by drawing a line in the middle of the GC layer. Then, we analyzed the number of $\mathrm{cFos}^{+}$ and tdTomato ${ }^{+}$neurons in each subdivisions of the rDG and cDG (Fig. 5C and D, respectively).

In the $\mathrm{rDG}$, significantly more $\mathrm{cFos}^{+}$neurons were located in the infrapyramidal than in the suprapyramidal blade during PSR than during W (Fig. 5C top left) (W vs PSR in infrapyramidal blade, $p=0.030$, suprapyramidal blade, $p=0.37$ ). There was also significantly more $\mathrm{cFos}^{+}$neurons during PSR than $\mathrm{W}$ in the core but not in the shell part of the rDG (Fig. 5C top right) (W vs PSR, core part, $p=0.024$, shell part, $\mathrm{p}=0.33$ ). Similarly, the number of tdTomato ${ }^{+}$neurons was higher during PSR than $\mathrm{W}$ in the infrapyramidal blade and the core part (Fig. 5C bottom left: W vs PSR in infrapyramidal blade, $p=0.036$, suprapyramidal blade, $p=0.10$ ).(Fig. $5 \mathrm{C}$ bottom right; $\mathrm{W}$ vs PSR, core part, $p=0.012$; shell part, $p=0.18$ ) We then performed the same analyzes for the cDG. Like for the rDG, there were significantly more $\mathrm{cFos}^{+}$neurons during PSR than $\mathrm{W}$ in the infrapyramidal but not in the suprapyramidal blade (W vs PSR, infrapyramidal blade, $p=0.042$; suprapyramidal blade, $p=0.26$ ). In contrast to the $\mathrm{rDG}$, they was no significant difference in the number of $\mathrm{cFos}^{+}$cells between PSR and $\mathrm{W}$ in the core and the shell parts of the DG, (W vs PSR, core: $p=0.33$; shell, $p=0.48$ ). Finally, there was no significant difference in the number of tdTomato ${ }^{+}$neurons between $\mathrm{W}$ and PSR in the two blades and parts of the cDG.

Then, we determined whether there was a positive correlation between the quantity of PS and the number of $\mathrm{cFos}^{+}$neurons in the two blades. We correlated the number of $\mathrm{cFos}^{+}$neurons with the amount of PS (\%) during the first $30 \mathrm{~min}$ of PSR (Fig. 1E, 5E). The number of $\mathrm{cFos}^{+}$neurons in the infrapyramidal (Fig. 5E left, red dots and line) but not in the suprapyramidal blade (blue dots and line) of the rDG was significantly and positively correlated with the amount of PS. Similarly, in the cDG (Fig. 5E right), the number of $\mathrm{cFos}^{+}$neurons in the infrapyramidal but not the suprapyramidal blade was also positively and significantly corelated with the amount of PS during PSR. Altogether, these data indicate that the neurons of the suprapyramidal blade of the DG are activated both during W and PSR while those located in the infrapyramidal blade are specifically activated during PSR.

\section{Discussion}


Here we showed that 1) the DG contained a large number of $\mathrm{cFos}^{+}$neurons during PSR in line with our previous report in rats using a different method of PSD 2) the number of $\mathrm{cFos}^{+}$neurons in the infrapyramidal blade of the DG was statistically correlated with PS quantities during PSR 3) Different GCs were activated when exposing successively the mice one week apart to two W, two PSR or a W and then a PSR. 4) In the SLD and SuM, the same neurons were reactivated when the mice were exposed twice to W or PSR but not to $\mathrm{W}$ and then PSR.

\subsection{Is cFos expression during PSR specifically induced in neurons activated during PS?}

The distribution and number of $\mathrm{cFos}^{+}$neurons reported in the SLD, SuM and the DG after PSR in the present study in mice was similar to that obtained in rats using the flowerpot PSD method ${ }^{4}$. We and others previously showed that corticosterone levels increase after PSD by the flowerpot method and that animals start to sleep after $30 \mathrm{~min}$ to $1 \mathrm{~h}$ of waking ${ }^{12,16}$. In contrast, with our new automatic PSD system $^{9,11}$ used in the present study, corticosterone levels were not increased and the mice immediately started to sleep after $\mathrm{PSD}^{11}$. Altogether, these results indicate that the presence of $\mathrm{cFos}^{+}$neurons in the SLD, SuM and DG during PSR is not species dependent and is not due to the stress induced by the PSD method and therefore likely corresponds to a specific activation during PS .

\subsection{TRAP method validation}

Recently, transgenic mice with either Tet-Off or CreER ${ }^{\mathrm{T} 2}$ system have been introduced to allow the permanent expression of transgenes in populations of neurons transiently expressing a specific protein like cFos after exposing the mice to a stimulus ${ }^{6,17,18}$. We chose the TRAP2 (cFos-CreER ${ }^{\mathrm{T} 2}$ ) model rather than the Tet-off model because we wanted to label (TRAP) neurons specifically activated during PSR. It has indeed been shown that when using 4-OHT, the expression of CreER ${ }^{\mathrm{T} 2}$ lasts less than $6 \mathrm{~h}^{7,8}$, while it lasts longer with the Tet-Off system due to the need to remove Dox for $24 \mathrm{~h}$. In our first study using the TRAP2 mice9 ${ }^{9}$, we showed that a substantial proportion of neurons labelled with tdTomato during W $(37.8 \%)$ or PSR $(14.4 \%)$ in the lateral hypothalamic area express cFos when the animals were re- 
exposed to the same conditions just before perfusion, whereas the proportions was inferior to $4 \%$ when the animals were exposed to two different conditions (W-PSR). Here, we also found that a large proportion of tdTomato ${ }^{+}$neurons in the SuM and the SLD were double-labeled in W-W (SuMM: 70\%) or PSR-PSR (SuML: $48.4 \%$, SLD:26.1\%) mice. In the hippocampus, $33 \%$ in cCA1 and $46.2 \%$ in rCA1 neurons were double-labeled in the W-W condition. Additionally, we found that the ratio of tdTomato ${ }^{+} \mathrm{cFos}^{+}$cells in W or PSR conditions was around 1 in the SLD, 0.5 in the SuM, 0.25 in DG and only of 0.1 in CA1. Such low TRAPing in CA1 was reported previously while high level of TRAPing was reported in other structures ${ }^{19}$. Further, it was previously reported in the thirst circuit that after water-deprivation, the percentage of double-labeled/total $\mathrm{cFos}^{+}$neurons was in the range of 0.5 1.0 while the percentage of double-labeled/total tdTomato ${ }^{+}$neurons was almost of $1.0^{7}$. In DeNardo et al. ${ }^{8}$, the ratio of tdTomato ${ }^{+} / \mathrm{cFos}^{+}$neurons was also around 0.4 in the prelimbic cortex when 4-OHT was injected after contextual fear conditioning (CFC)-training and mice were perfused after CFC-test. These and our results indicate that the level of tdTomato expression is close to that of cFos in most subcortical structures while it is lower but still high in most cortical structures. The mechanisms underlying the variability in the efficiency of TRAPing across structures is unclear but could be due to the type of neurons, the level of transgene expression or the local availability of 4-OHT. In conclusion, our and previous results indicate that the TRAP method is efficient and can be used to map neurons expressing cFos.

\subsection{Different neurons are activated during $W$ and PS in the SUM and SLD.}

Our previous ${ }^{4}$ and present results confirm that the SuML is more activated during PSR than the SuMM. In agreement with previous studies ${ }^{20,21}$, we also observed in the PSR-PSR mice, a substantial number of tdTomato ${ }^{+}$and $\mathrm{cFos}^{+}$neurons in the SLD, a key structure for inducing PS. Further, we show for the first time that most of the SuM and SLD neurons are reactivated when the mice are exposed to the same condition one week later, while different neurons are activated during W and PSR. Our results open the possibility to study in unprecedented detail the role of SLD and SuM neurons during W and PS by combining the TRAP method with calcium imaging, opto, chemogenetic, and tracing tools. 


\subsection{Differential activation of the Hippocampus (HIP) during $W$ and PSR}

The distribution of $\mathrm{cFos}^{+}$neurons in the rHIP is in line with our previous data obtained in rats ${ }^{4}$. In addition, we report for the first time the distribution of cFos + neurons in the cHip. In agreement with our results obtained during $\mathrm{W}$, it has been previously shown that CA1 and the suprapyramidal but not the infrapyramidal blade of DG are activated in mice exposed to a novel environment, spatial memory, socialization, and stress ${ }^{22-26}$. Additionally, we found in the present study that the large number of GCs labeled in the DG during PSR were similarly distributed over the two blades. Further, the number of $\mathrm{cFos}^{+}$GCs in the infrapyramidal but not the suprapyramidal blade during PSR was statistically and positively correlated with the amount of PS. To our knowledge such results have never been reported before. To identify the function of such activation during PS, it will be crucial in the future to determine whether GCs of the infrapyramidal blade are activated during $\mathrm{W}$ in some conditions not yet explored.

Concerning reactivation, the finding that a large number of cells in CA1 are double-labeled when exposing the mice twice one week apart to the same open field (the W-W group) fits with previous electrophysiological studies showing that place cells of $\mathrm{CA} 1$ are reactivated when the mice are put again in the same environment ${ }^{27-32}$. It is also in line with studies using TRAP or Tet-Off mice showing a high percentage of double-labeled cells in CA1 when exposing mice to the same task or environment one or several days apart ${ }^{33,34}$.

Our results showing the absence of reactivation in the DG in all groups of mice are more puzzling. Indeed, it has been reported that 2 to $10 \%$ of the GCs in the DG activated during a learning task form a memory engram, and are reactivated during a retrieval test performed 2-5 days later ${ }^{33,35,36}$. Further, Kumar et al. recently showed that $38 \%$ of 4 weeks adult born GCs of the suprapyramidal blade activated during a CFC are reactivated during subsequent PS episodes occurring during the first $4 \mathrm{~h}$ after exposing the mice to the $\mathrm{CFC}^{37}$. Our negative results might therefore be due to the fact that the exposure to the same condition (W-W, PSR-PSR) or to a PSR after W occurred one week apart, and that with such longtime gap, GCs belonging to different memory engrams are activated in the DG. 
We previously demonstrated that the activation of GCs in the DG during PSR is induced by a projection from SuML neurons ${ }^{4}$. Here we showed that the same SuML neurons are reactivated one week apart during PSR while different GCs are reactivated in the DG. Using optogenetic stimulation of terminals of SuML neurons in the DG, we recently showed that all GCs are both excited and inhibited by the SuML projection ${ }^{38}$. It suggests that although the SuML neurons excite all GCs during PS, other input(s) like the performant path ${ }^{39}$ is (are) likely necessary to induce the activation of different GCs over two periods of PSR separated by one week. Based on our and Kumar et al. results ${ }^{37}$, it can be postulated that reactivation of the same GCs during PS might occur only during a few hours while different cells belonging to different memory engrams would be activated after one week.

Acknowledgements: This work is supported by CNRS (UMR5292), INSERM (U1028), SFRMS, and University Lyon1. This work was also supported by Japan Society for the Promotion of Science, The Uehara Memorial Foundation, and The French embassy in Japan (to R.Y.), and China Scholarship Council (to D-R. W.). We thank Dr. Liqun Luo (Stanford University, CA) for providing us TRAP2 mice.

\section{Disclosure Statement}

The authors declare no competing financial interests. 


\section{References}

1. Jouvet M. and Michel F. (1959) [Electromyographic correlations of sleep in the chronic decorticate \& mesencephalic cat].C R Seances Soc Biol Fil. 153:422-425

2. Buzsáki G. (2002) Theta oscillations in the hippocampus. Neuron. 33:325-340.

3. Montgomery SM, Sirota A, Buzsáki G. (2008) Theta and gamma coordination of hippocampal networks during waking and rapid eye movement sleep. J Neurosci. 28:6731-6741.

4. Renouard L, Billwiller F, Ogawa K, et al. The supramammillary nucleus and the claustrum activate the cortex during REM sleep. Sci Adv. (2015) 1:e1400177.

5. Billwiller F, Renouard L, Clement O, Fort P, Luppi PH. Differential origin of the activation of dorsal and ventral dentate gyrus granule cells during paradoxical (REM) sleep in the rat. Brain Struct Funct. (2017) 222:1495-1507.

6. Guenthner CJ, Miyamichi K, Yang HH, Heller HC, Luo L. (2013) Permanent genetic access to transiently active neurons via TRAP: targeted recombination in active populations. Neuron. 78:773-784.

7. Allen WE, DeNardo LA, Chen MZet al. Thirst-associated preoptic neurons encode an aversive motivational drive. Science. (2017) 357:1149-1155.

8. DeNardo LA, Liu CD, Allen WE et al. Temporal evolution of cortical ensembles promoting remote memory retrieval. Nat Neurosci. (2019) 22:460-469.

9. Lee HS, Yamazaki R, Wang D, et al. Targeted recombination in active populations as a new mouse genetic model to study sleep-active neuronal populations: Demonstration that Lhx6+ neurons in the ventral zona incerta are activated during paradoxical sleep hypersomnia. J Sleep Res. (2020):e12976.

10. Libourel PA, Corneyllie A, Luppi PH, Chouvet G, Gervasoni D. (2015) Unsupervised online classifier in sleep scoring for sleep deprivation studies. Sleep. 38:815-828. 
11. Arthaud S, Libourel PA, Luppi PH, Peyron C. Insights into paradoxical (REM) sleep homeostatic regulation in mice using an innovative automated sleep deprivation method. Sleep. (2020) 43:zsaa003.

12. Arthaud S, Varin C, Gay N, et al. Paradoxical (REM) sleep deprivation in mice using the smallplatforms-over-water method: polysomnographic analyses and melanin-concentrating hormone and hypocretin/orexin neuronal activation before, during and after deprivation. J Sleep Res. (2015) 24:309319.

13. Boissard R, Gervasoni D, Schmidt MH, Barbagli B, Fort P, Luppi PH. The rat ponto-medullary network responsible for paradoxical sleep onset and maintenance: a combined microinjection and functional neuroanatomical study. Eur J Neurosci. (2002) 16:1959-1973.

14. Harland B, Contreras M, and Fellous J-M. A Role for the Longitudinal Axis of the Hippocampus in Multiscale Representations of Large and Complex Spatial Environments and Mnemonic Hierarchies. The Hippocampus-Plasticity and Functions: IntechOpen; 2018.

15. Martin LA, Tan SS, and Goldowitz D. (2002) Clonal architecture of the mouse hippocampus. J Neurosci. 22:3520-3530.

16. Mehta R, Khan S, and Mallick BN. (2018) Relevance of deprivation studies in understanding rapid eye movement sleep. Nat Sci Sleep. ;10:143-158.

17. Liu X, Ramirez S, Pang PT, et al. (2012) Optogenetic stimulation of a hippocampal engram activates fear memory recall. Nature. 484:381-385.

18. DeNardo L and Luo L. Genetic strategies to access activated neurons. Curr Opin Neurobiol. (2017) 45:121-129.

19. Deng W, Mayford M, Gage FH. Selection of distinct populations of dentate granule cells in response to inputs as a mechanism for pattern separation in mice. Elife. (2013) 2:e00312.

20. Clément O, Sapin E, Bérod A, Fort P, Luppi PH.Evidence that neurons of the sublaterodorsal tegmental nucleus triggering paradoxical (REM) sleep are glutamatergic. Sleep. (2011) 34:419-423. 
21. Valencia Garcia S, Libourel PA, Lazarus M, et al.: Genetic inactivation of glutamate neurons in the rat sublaterodorsal tegmental nucleus recapitulates REM sleep behaviour disorder. Brain. 140:414-428., 2017

22. Chawla MK, Guzowski JF, Ramirez-Amaya V et al. Sparse, environmentally selective expression of Arc RNA in the upper blade of the rodent fascia dentata by brief spatial experience. Hippocampus. (2005) 15:579-586.

23. Chawla MK, Sutherland VL, Olson K, McNaughton BL, Barnes CA. Behavior-driven arc expression is reduced in all ventral hippocampal subfields compared to CA1, CA3, and dentate gyrus in rat dorsal hippocampus.Hippocampus. (2018) 28:178-185.

24. Penke Z, Chagneau C, Laroche S. (2011) Contribution of Egr1/zif268 to Activity-Dependent Arc/Arg3.1 Transcription in the Dentate Gyrus and Area CA1 of the Hippocampus. Front Behav Neurosci. 5:48.

25. Gallitano AL, Satvat E, Gil M, Marrone DF. Distinct dendritic morphology across the blades of the rodent dentate gyrus. Synapse. (2016) 70:277-282.

26. Erwin SR, Sun W, Copeland M, Lindo S, Spruston N, Cembrowski MS. A Sparse, Spatially Biased Subtype of Mature Granule Cell Dominates Recruitment in Hippocampal-Associated Behaviors. Cell Rep. (2020) 31:107551.

27. O'Keefe J, Dostrovsky (1971) The hippocampus as a spatial map. Preliminary evidence from unit activity in the freely-moving rat. J.Brain Res. 34:171-175.

28. Muller RU, Kubie JL, Ranck JB Jr. (1987) Spatial firing patterns of hippocampal complex-spike cells in a fixed environment. J Neurosci. 7:1935-1950.

29. Smith DM and Mizumori SJ. (2006) Hippocampal place cells, context, and episodic memory. Hippocampus. 16:716-729.

30. Takahashi S. (2013) Hierarchical organization of context in the hippocampal episodic code. Elife. 2:e00321. 
31. Takahashi S. (2015) Episodic-like memory trace in awake replay of hippocampal place cell activity sequences. Elife. 4:e08105.

32. Tanaka KZ, He H, Tomar A, Niisato K, Huang AJY, McHugh TJ. (2018) The hippocampal engram maps experience but not place. Science. 361:392-397.

33. Tayler KK, Tanaka KZ, Reijmers LG, Wiltgen BJ. (2013) Reactivation of neural ensembles during the retrieval of recent and remote memory. Curr Biol. 23:99-106.

34. Okuyama T, Kitamura T, Roy DS, Itohara S, Tonegawa S. Ventral CA1 neurons store social memory. Science. (2016) 353:1536-1541.

35. Denny CA, Kheirbek MA, Alba EL,et al. (2014) Hippocampal memory traces are differentially modulated by experience, time, and adult neurogenesis. Neuron. 83:189-201.

36. Cazzulino AS, Martinez R, Tomm NK, Denny CA. (2016) Improved specificity of hippocampal memory trace labeling. Hippocampus. 26:752-762.

37. Kumar D, Koyanagi I, Carrier-Ruiz A, et al. (2020) Sparse Activity of Hippocampal Adult-Born Neurons during REM Sleep Is Necessary for Memory Consolidation. Neuron. 107:552-565.e10.

38. Billwiller F, Castillo L, Elseedy H, et al. GABA-glutamate supramammillary neurons control theta and gamma oscillations in the dentate gyrus during paradoxical (REM) sleep. Brain Struct Funct. (2020) doi: 10.1007/s00429-020-02146-y.

39. Hashimotodani Y, Karube F, Yanagawa Y, Fujiyama F, Kano M. (2018) Supramammillary Nucleus Afferents to the Dentate Gyrus Co-release Glutamate and GABA and Potentiate Granule Cell Output. Cell Rep. 25:2704-2715.e4. 


\section{Figure Legends}

\section{Figure1: TRAPing experimental design and paradoxical sleep rebound (PSR) protocol}

(A) Principle and scheme of TRAP method. (B) Experimental design with time course. The first condition (W or PSR) is paired with an injection of 4-OHT (50 mg/kg, i.p.) to label the neurons activated (TRAPing). One week later, the same mice were exposed to a second condition (W or PSR). To obtain cFos expression in the neurons activated during the second condition, the animals were deeply anesthetized and perfused $2 \mathrm{~h}$ after the exposure to the 2 nd condition. See "Materials and Methods" for the detailed information on each condition. (C) Representative EEG and EMG signals during each vigilance state. (D) The amounts of each vigilance state (\%) during $2 \mathrm{~h}$ of PSR (green bars) or $2 \mathrm{~h}$ of control recordings in the same mice between 10:00 and 12:00 (Ctrl, white bars). Black line, median; black dots, individual values; boxes, first and last quartiles; whiskers, minimum and maximum values excluding outliers. Significance Wilcoxon Signed-Rakes test $(\mathrm{n}=8) \cdot p<0.001^{* *}(\mathrm{E}-\mathrm{G})$ Amount $(\%)$ of PS (E), W(F) and SWS (G) for each 30-min bin during 2h of PSR (Green line). The data for Ctrl condition (black line) were obtained the day before PS deprivation. Significance Wilcoxon SignedRanks test (n=8). $p<0.05^{*}, p<0.001^{* *}(\mathrm{H})$ Immunofluorescence staining of tdTomato (red) and cFos (green) in SLD in a PSR-PSR mice.

\section{Figure 2: Different neurons are activated in the SuM during $W$ and PSR}

(A, B) Number of $\mathrm{cFos}^{+}$(A) and $\operatorname{tdTomato}^{+}$(B) neurons during either $\mathrm{W}(\mathrm{n}=3$ from the $\mathrm{W}-\mathrm{W}$ group, blue bars) or PSR ( $\mathrm{n}=8$ from the PSR-PSR and W-PSR groups, green bars) in SuMM and SuML. Significance Wilcoxon Signed-Ranks test when comparing SuMM and SuML and Mann-Whitney's U test when comparing between $\mathrm{W}$ and PSR $(\mathrm{n}=8) . p<0.05^{*}, p<0.01^{* *}$. (C-E) Immunofluorescence staining of tdTomato (red) and cFos (green) from representative sections in SuM from the W-W (C), PSR-PSR (D), and W-PSR (E) groups. (F) Percentage of reactivations $\left(\%=\right.$ tdTomato ${ }^{+} / \mathrm{cFos}^{+}$neurons over total number of tdTomato ${ }^{+}$neurons $* 100$ ) by group are shown. Black line, median; black dots, 
individual values; boxes, first and last quartiles; whiskers, minimum and maximum values excluding outliers. Significance Kruskal-Wallis followed by a Dunn's multiple comparison test. $p<0.05^{*}$ and $p<0.01^{* *}$ vs W-W, $p<0.05^{\#}$ vs PSR-PSR. MM; mammillary body, mt; mammillary tract, SuML and SuMM; supramammillary nucleus, lateral and medial part. Bars $=50 \mu \mathrm{m}$

\section{Figure 3: Distribution of tdTomato and cFos ${ }^{+}$neurons in the rostral hippocampus}

(A-F) Immunostaining of tdTomato (brown) and cFos (black) in representative mice and schematic drawings of sections depicting the location of tdTomato ${ }^{+}$(red), $\mathrm{cFos}^{+}$(green), and $\mathrm{tdTomato}^{+} / \mathrm{cFos}^{+}$ (black) neurons in rHPC from W-W (A and B), PSR-PSR (C and D), and W-PSR (E and F) mice are shown. (G) Number of cFos + neurons during either $\mathrm{W}$ ( $\mathrm{n}=3$ from the $\mathrm{W}-\mathrm{W}$ group, blue bars) or PSR ( $\mathrm{n}=8$ from the PSR-PSR and W-PSR groups, green bars) in rCA1, rCA3, and rDG. Significance MannWhitney's U test when comparing between W and PSR. $p<0.05 *(\mathrm{H})$ Percentage of reactivations $(\%=$ $\mathrm{tdTomato}^{+} / \mathrm{cFos}^{+}$neurons over total tdTomato ${ }^{+}$neurons $* 100$ ) for each structure. Significance KruskalWallis followed by a Dunn's multiple comparison test. $p<0.05^{*}$ vs W-W. Black line, median; black dots, individual values; boxes, first and last quartiles; whiskers, minimum and maximum values excluding outliers. DG; dentate gyrus

\section{Figure 4: Distribution of tdTomato and cFos+ neurons in the caudal hippocampus}

(A-F) Immunostaining of tdTomato (brown) and cFos (black) from representative mice and schematic drawings of sections depicting the location of tdTomato ${ }^{+}$(red), $\mathrm{cFos}^{+}$(green), and tdTomato $/ \mathrm{cFos}^{+}$ (black) neurons in cHPC from W-W (A and B), PSR-PSR (C and D), and W-PSR (E and F) mice are shown. (G) Number of $\mathrm{cFos}^{+}$neurons during either $\mathrm{W}$ ( $\mathrm{n}=3$ from the $\mathrm{W}-\mathrm{W}$ group, blue bars) or PSR ( $\mathrm{n}=8$ from the PSR-PSR and W-PSR groups, green bars) in rCA1, rCA3, and rDG. Significance MannWhitney's U test when comparing between W and PSR. $p<0.05^{*}, p<0.01 * *$ (H) Percentage of reactivations $\left(\%=\right.$ tdTomato $^{+} / \mathrm{cFos}^{+}$neurons over total tdTomato ${ }^{+}$neurons $\left.* 100\right)$. Significance KruskalWallis followed by a Dunn's multiple comparison test. $p<0.05^{*}$ vs W-W. Black line, median; black dots, 
individual values; boxes, first and last quartiles; whiskers, minimum and maximum values excluding outliers.

\section{Figure 5: The number of activated neurons in the DG is positively correlated with the amount of PS}

(A) Scheme of the granule cell layer (GCL) of the DG and its subdivisions. Suprapyramidal and infrapyramidal blades were further divided into shell and core parts. The red line is the crest (i.e. limit between suprapyramidal and infrapyramidal blades). (B) Immunofluorescence of tdTomato (red) and cFos (green) in rDG from one representative mice per group (C and D) The number of $\mathrm{cFos}^{+}$(top) and tdTomato $^{+}$(bottom) neurons in the rDG (C) and cDG (D) are shown. Graphs on the left show the number of labeled neurons in each (infrapyramidal or suprapyramidal) blade. Graphs on the right show the number of labeled neurons in the shell and core parts. Black line, median; black dots, individual values; boxes, first and last quartiles; whiskers, minimum and maximum values excluding outliers. Significance Wilcoxon Signed-Ranks test when comparing infrapyramidal blade and suprapyramidal blade or core and shell inside the same group (i.e. comparison between the same-color bars), $p<0.05^{*}$. MannWhitney's U test was used when comparing between W and PSR, $p<0.05^{\#}, p<0.01^{\# \#}$ (E) Correlation between the amount of PS (\% during the 30-60 min of PSR, horizontal axis) and the number of $\mathrm{cFos}^{+}$ neurons in the entire DG (black), the suprapyramidal (red) and infrapyramidal blades (blue) (vertical axis) in the rDG (left) and cDG (right) $(\mathrm{n}=11)$. Pearson's R values $(r)$ and their probabilities are as follows: total ( $\mathrm{rDG}, r=0.72, p=0.0059$; $\mathrm{cDG}, \mathrm{r}=0.42, p=0.12$ ), suprapyramidal ( $\mathrm{rDG}, r=0.30, p=0.19$; cDG, -0.073, $p=0.42$ ), and infrapyramidal (rDG, $r=0.85, p<0.001$; $\mathrm{cGD}, \mathrm{r}=0.76, p=0.0033$ ). 


\begin{tabular}{|c|c|c|c|c|c|c|c|c|c|c|c|c|}
\hline \multirow[b]{2}{*}{ Region } & \multicolumn{4}{|c|}{ Wake/Wake } & \multicolumn{4}{|c|}{ PSR/PSR } & \multicolumn{4}{|c|}{ Wake/PSR } \\
\hline & $\begin{array}{c}\text { Total } \\
\text { TRAP } \\
\end{array}$ & $\begin{array}{l}\text { Total } \\
\text { cFos }\end{array}$ & Double & $\begin{array}{c}\% \text { Double } \\
\text { /TRAP }\end{array}$ & $\begin{array}{c}\text { Total } \\
\text { TRAP } \\
\end{array}$ & $\begin{array}{l}\text { Total } \\
\text { cFos }\end{array}$ & Double & $\begin{array}{c}\% \text { Double } \\
\text { /TRAP }\end{array}$ & $\begin{array}{c}\text { Total } \\
\text { TRAP } \\
\end{array}$ & $\begin{array}{l}\text { Total } \\
\text { cFos }\end{array}$ & Double & $\begin{array}{c}\% \text { Double } \\
\text { /TRAP }\end{array}$ \\
\hline \multirow{2}{*}{ rCA1 } & 3.0 & 129.0 & 1.0 & 33.3 & 7.0 & 5.0 & 0.0 & 0.0 & 8.0 & 5.0 & 0.0 & 0.0 \\
\hline & 3.0 & 107.5 & 0.5 & 5.6 & 3.5 & 5.5 & 0.5 & 5.6 & 9.5 & 15.5 & 1.0 & 7.1 \\
\hline \multirow{2}{*}{ cCA1 } & 34.0 & 530.0 & 20.0 & 46.2 & 31.0 & 65.0 & 1.0 & 3.2 & 81.0 & 53.0 & 3.0 & 2.9 \\
\hline & 22.5 & 303.5 & 11.5 & 9.7 & 20.5 & 42.0 & 3.5 & 5.8 & 26.0 & 35.0 & 1.0 & 1.3 \\
\hline \multirow{2}{*}{ rCA3 } & 4.0 & 60.0 & 0.0 & 0.0 & 4.5 & 9.0 & 0.5 & 10.0 & 6.0 & 11.0 & 0.0 & 0.0 \\
\hline & 4.5 & 27.0 & 0.0 & 0.0 & 4.5 & 9.5 & 0.5 & 5.6 & 4.0 & 17.5 & 0.0 & 0.0 \\
\hline \multirow{2}{*}{ cCA3 } & 4.0 & 52.0 & 0.0 & 0.0 & 1.0 & 3.0 & 0.0 & 0.0 & 3.5 & 4.0 & 0.0 & 0.0 \\
\hline & 2.0 & 15.0 & 0.5 & 8.3 & 0.0 & 7.0 & 0.0 & 0.0 & 6.0 & 11.5 & 0.0 & 0.0 \\
\hline \multirow{2}{*}{$\mathrm{rDG}$} & 8.0 & 63.0 & 0.0 & 0.0 & 26.0 & 100.0 & 0.0 & 0.0 & 17.0 & 80.0 & 0.0 & 0.0 \\
\hline & 11.0 & 8.0 & 0.0 & 0.0 & 9.5 & 9.0 & 0.3 & 0.6 & 16.5 & 23.0 & 0.5 & 0.0 \\
\hline \multirow{2}{*}{$\mathrm{cDG}$} & 8.0 & 73.0 & 0.0 & 0.0 & 14.5 & 53.0 & 0.5 & 3.4 & 12.0 & 52.0 & 0.0 & 0.0 \\
\hline & 16.5 & 46.5 & 2.3 & 5.8 & 5.5 & 35.5 & 0.5 & 2.3 & 10.0 & 49.0 & 1.0 & 0.0 \\
\hline \multirow{2}{*}{ SuMM } & 25.0 & 53.0 & 17.5 & 70.0 & 10.0 & 19.5 & 4.0 & 23.5 & 14.5 & 16.0 & 2.0 & 13.8 \\
\hline & 5.5 & 9.5 & 7.0 & 13.1 & 5.3 & 0.8 & 1.8 & 17.0 & 5.0 & 5.5 & 2.0 & 14.3 \\
\hline \multirow{2}{*}{ SuML } & 32.5 & 51.0 & 14.0 & 50.0 & 22.0 & 48.0 & 12.0 & 48.4 & 31.0 & 34.0 & 7.0 & 19.4 \\
\hline & 16.0 & 19.5 & 13.3 & 14.6 & 8.5 & 15.0 & 3.8 & 4.2 & 17.0 & 39.5 & 3.0 & 10.6 \\
\hline \multirow{2}{*}{ SLD } & \multirow{2}{*}{\multicolumn{4}{|c|}{ N.A. }} & 24.0 & 20.0 & 6.0 & 26.1 & 14.0 & 30.5 & 1.5 & 10.5 \\
\hline & & & & & 1.0 & 2.0 & 1.0 & 4.6 & 3.0 & 10.5 & 0.5 & 1.3 \\
\hline
\end{tabular}

Table 1 Number of tdTomato ${ }^{+}\left(\right.$TRAP), cFos $^{+}$, double-labeled neurons (tdTomato ${ }^{+}$and cFos $\left.^{+}\right)$, and percentage of reactivated neurons $(\%=$ double/tdTomato*100) by structure in the three groups of mice.

For each structure, the median number of neurons (top) and the interquartile range (IQR) (bottom) are displayed. The total number of TRAP (tdTomato + ) and $\mathrm{cFos}^{+}$neurons given correspond to the sum of the single (either tdTomato ${ }^{+}$or $\mathrm{cFos}^{+}$) and double (tdTomato ${ }^{+}$and $\mathrm{cFos}^{+}$) labeled neurons in the W-W $(\mathrm{N}=3)$, PSR-PSR $(\mathrm{N}=3)$, and $\mathrm{W}-\mathrm{PSR}(\mathrm{N}=5)$ groups. 
A
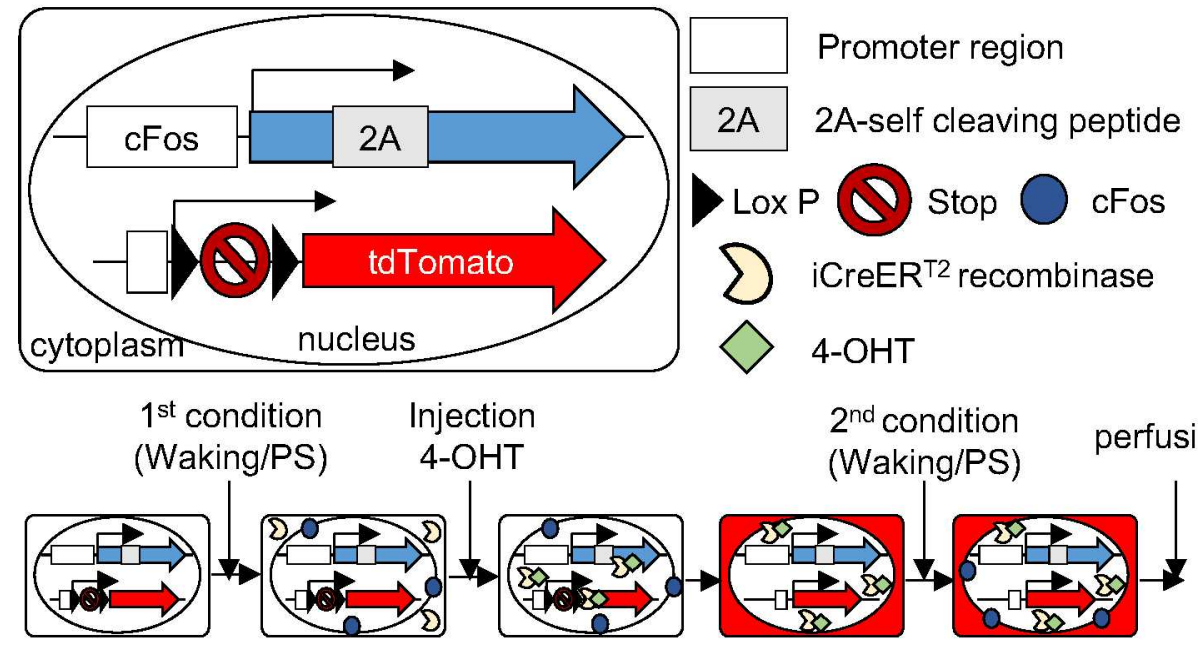

B
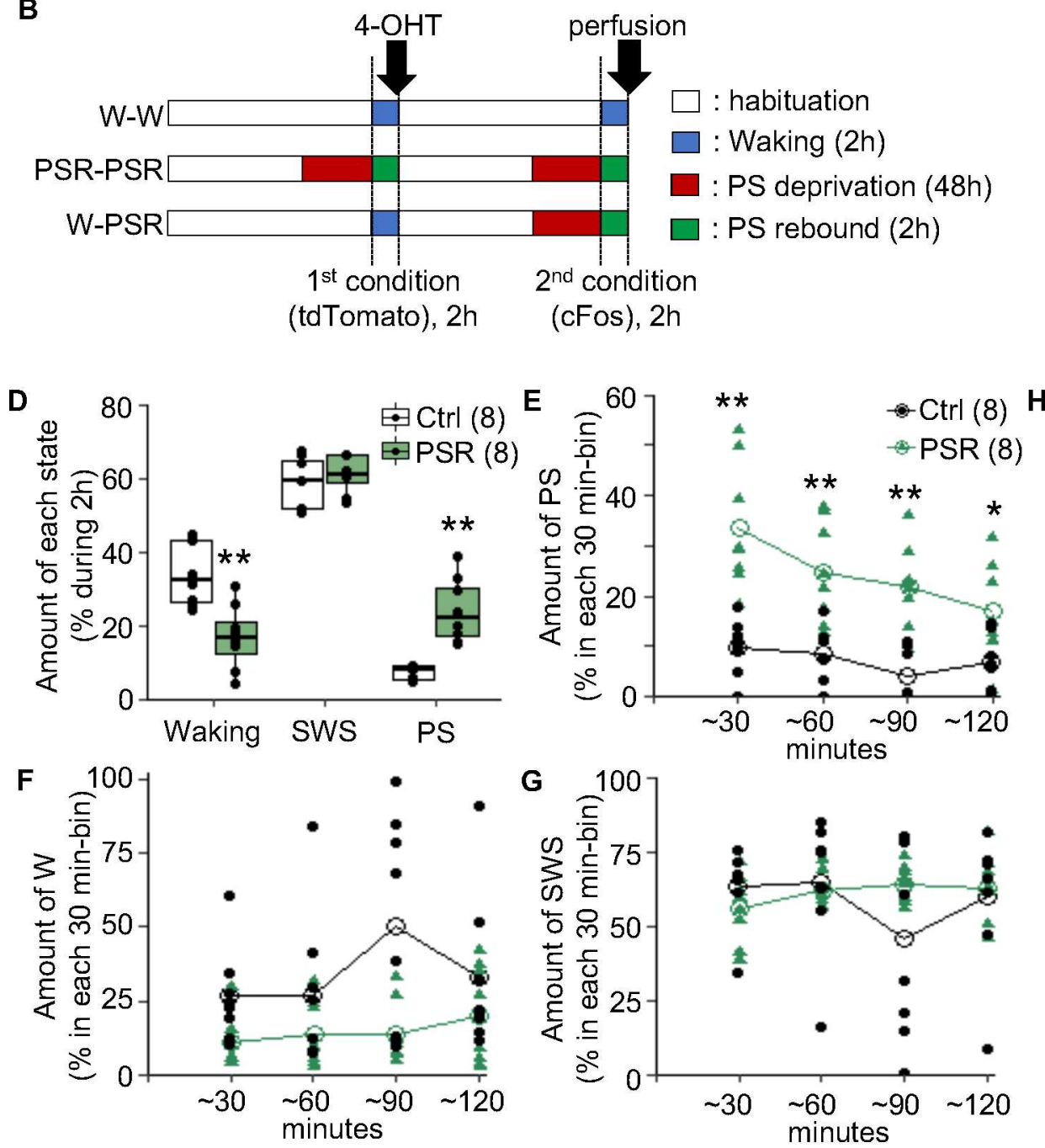

C

Waking

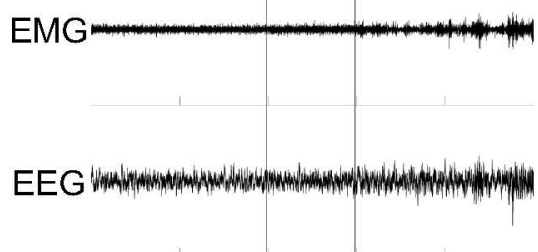

SWS

EMG

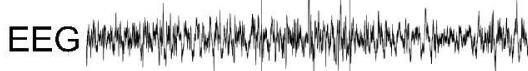

PS
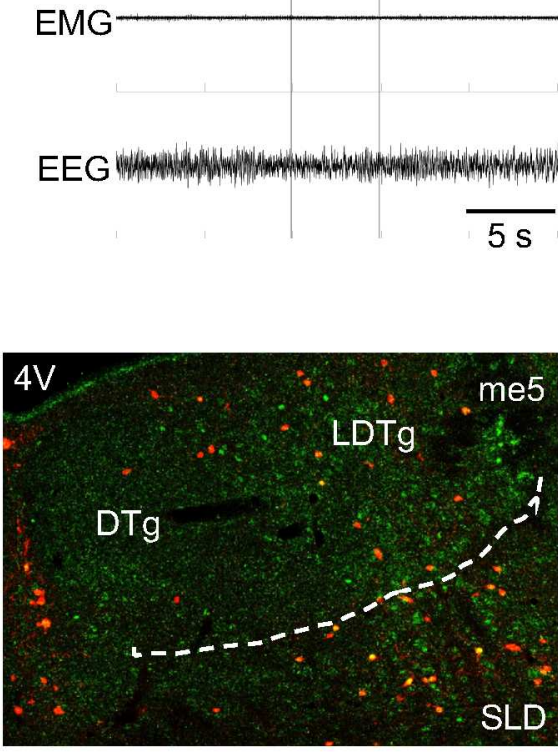

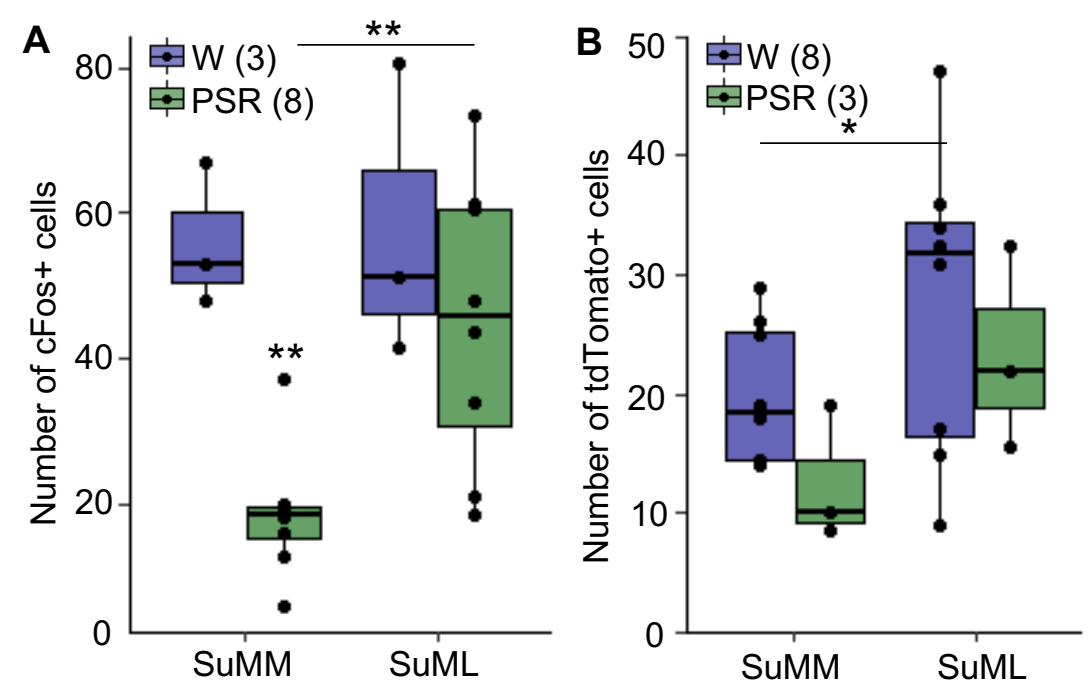

C

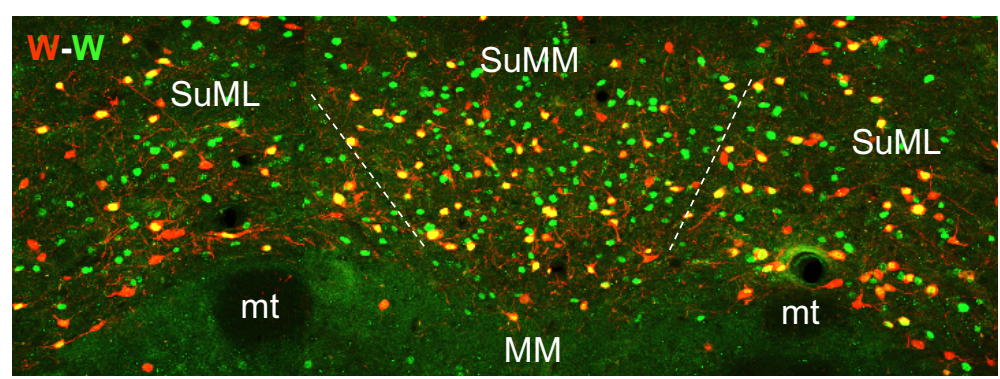

D

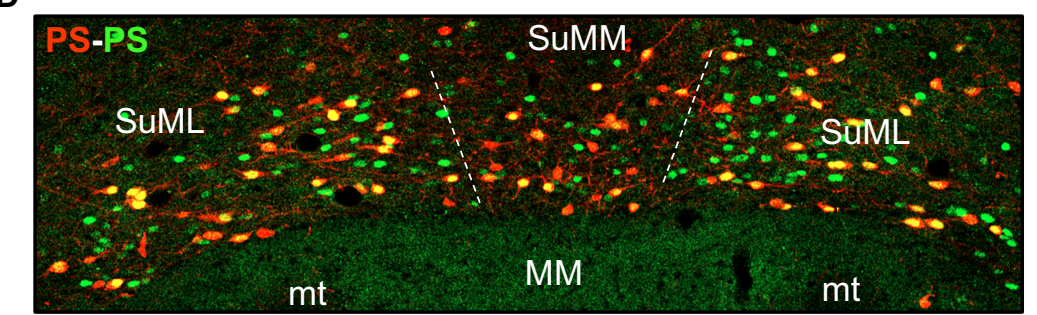

E

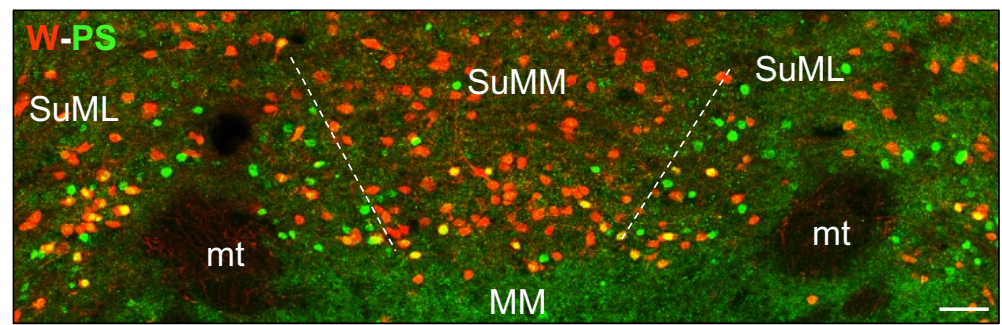

43
44
45
46
47
48
49
50
51
52
53
54
55
56
57
58
59
60

(2)

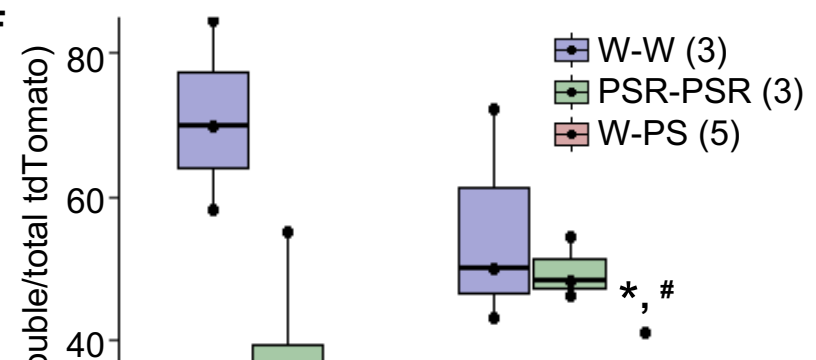

https://mc.m\&4MAMAiptcentral.conßjystep 
B
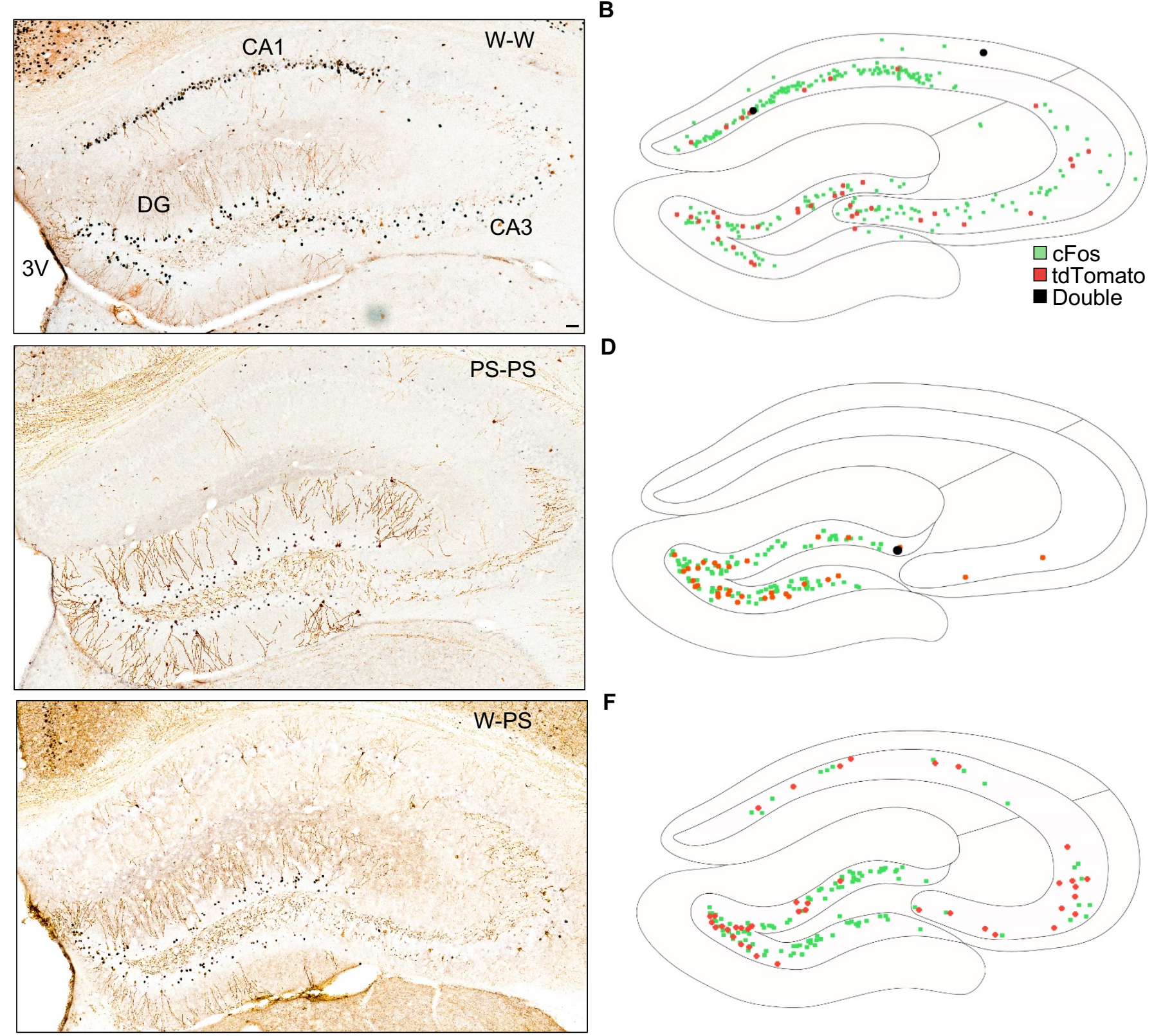

$F$

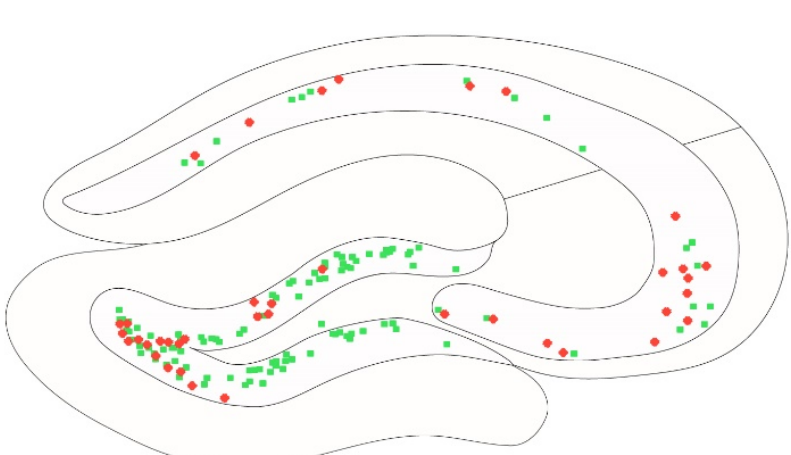

40

4

H
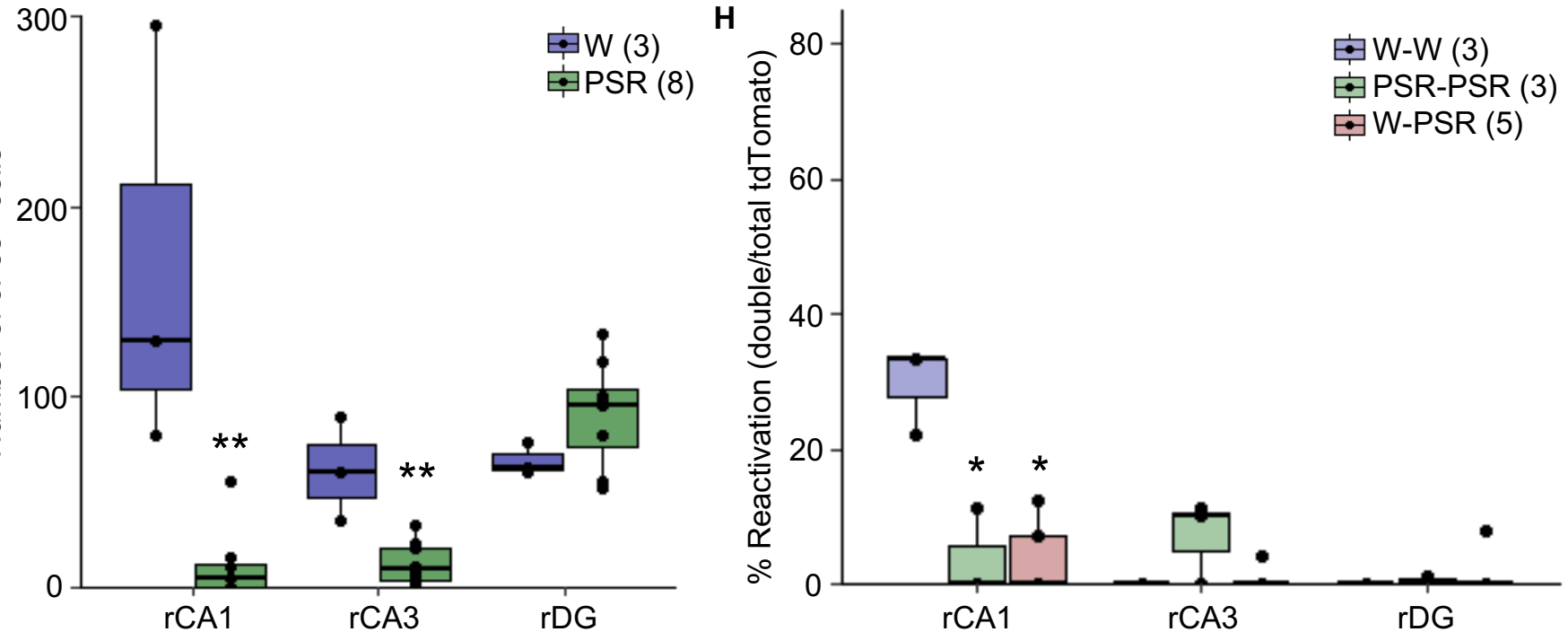

https://mc.manuscriptcentral.com/jsleep

Figure 3 


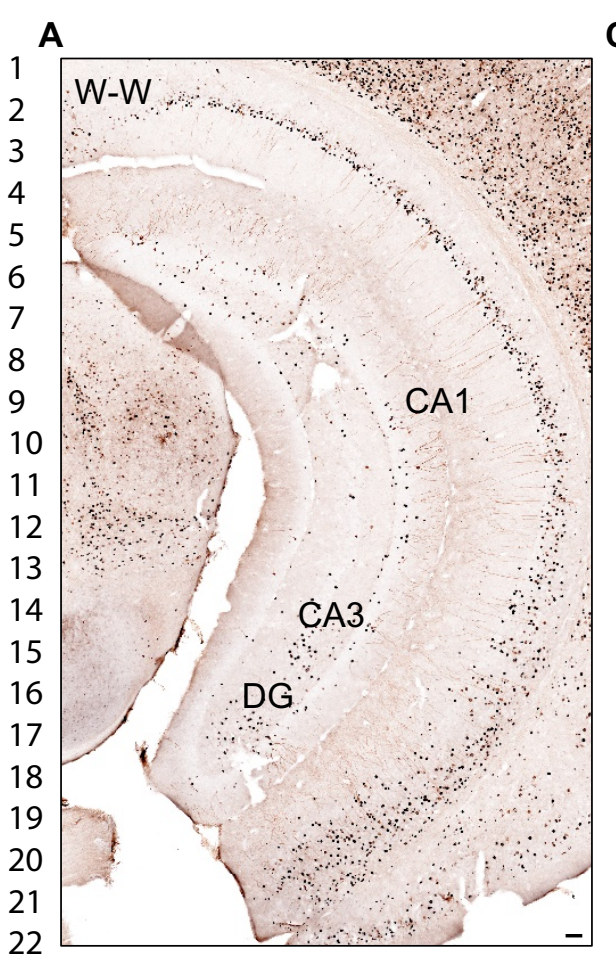

C

E
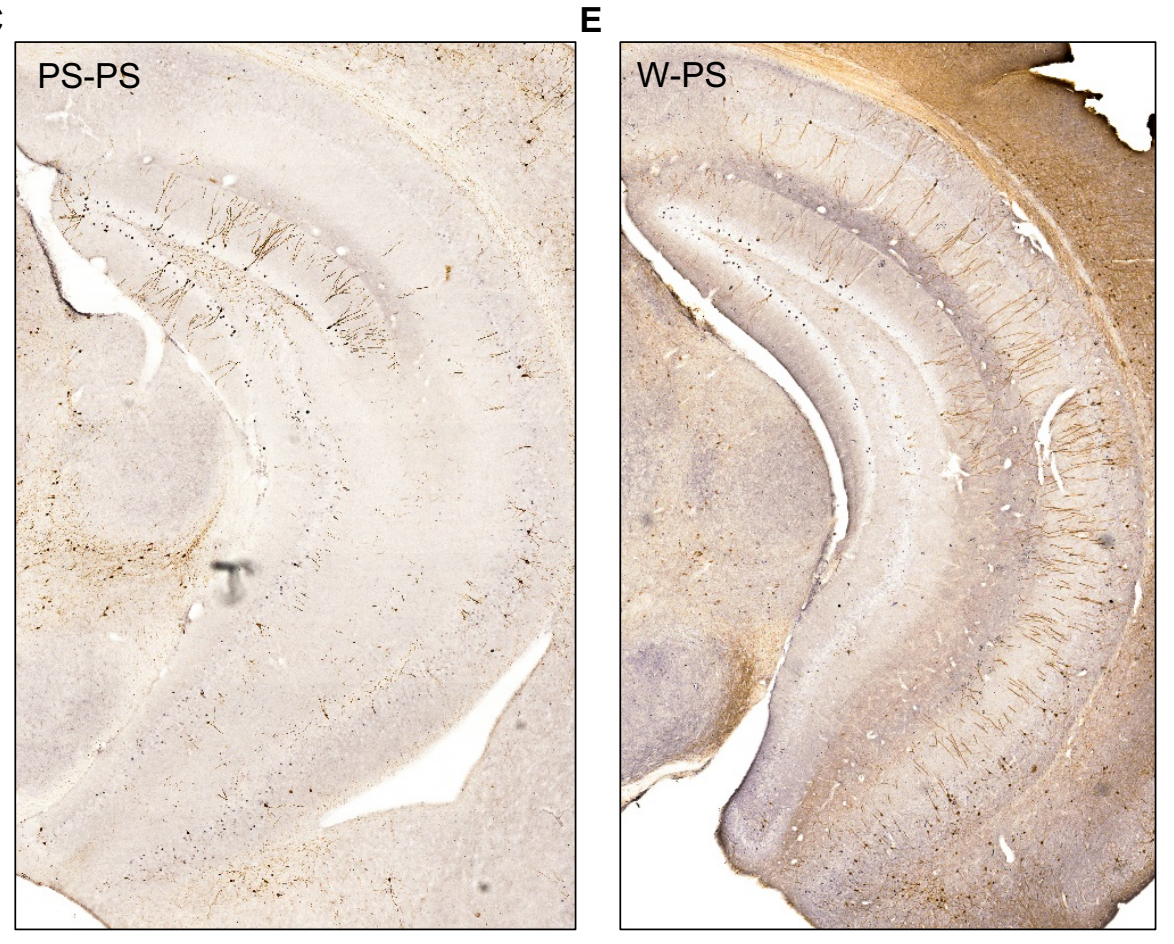

23

D

F
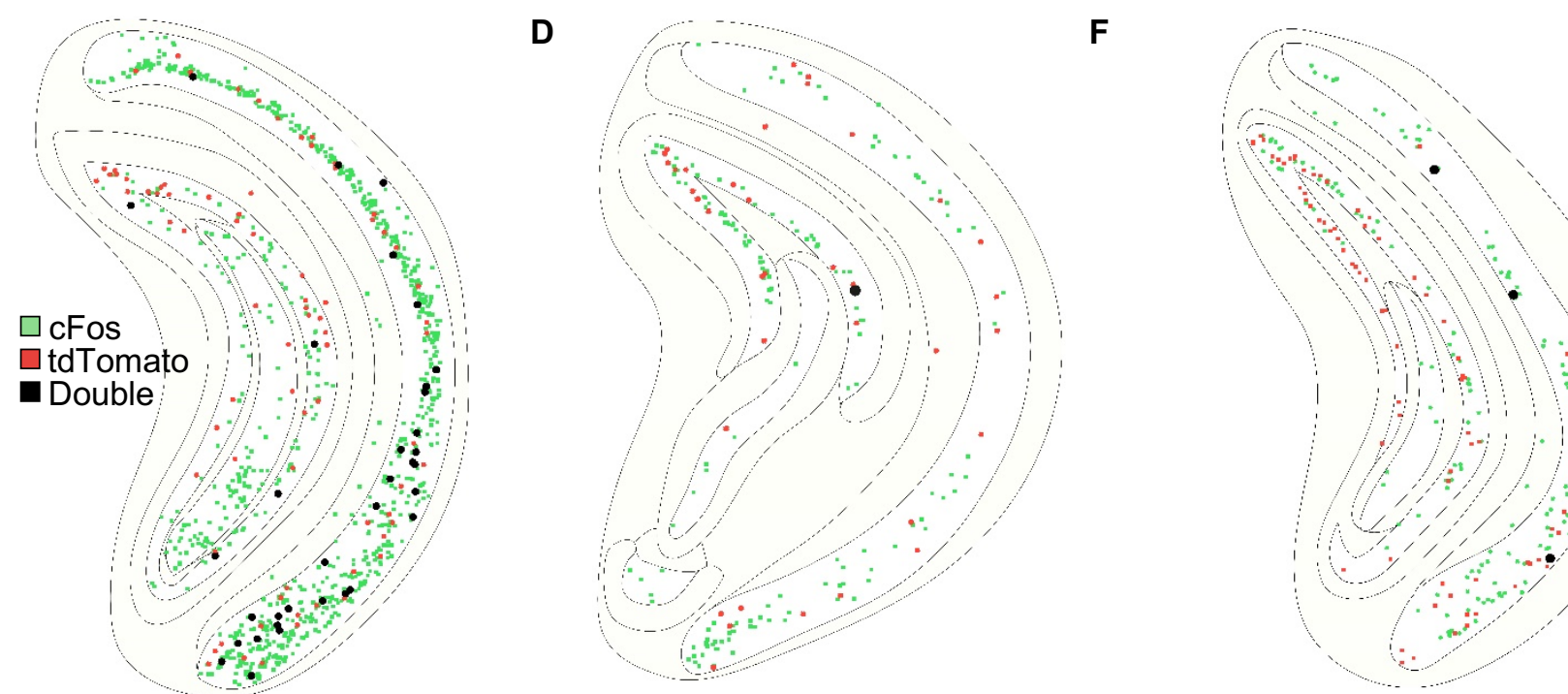

40

$41^{4}$

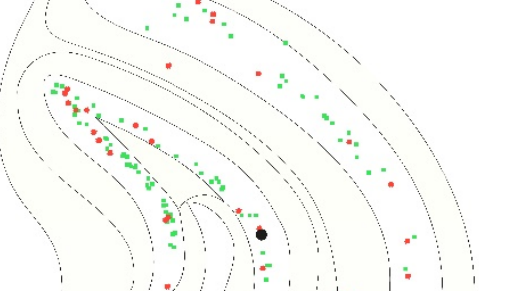

W-W (3)

PSR-PSR (3)

E W-PSR (5)

42
43

43

44

$45 \stackrel{0}{0}$

468

$47+$

48 난

4

5

51

52

5

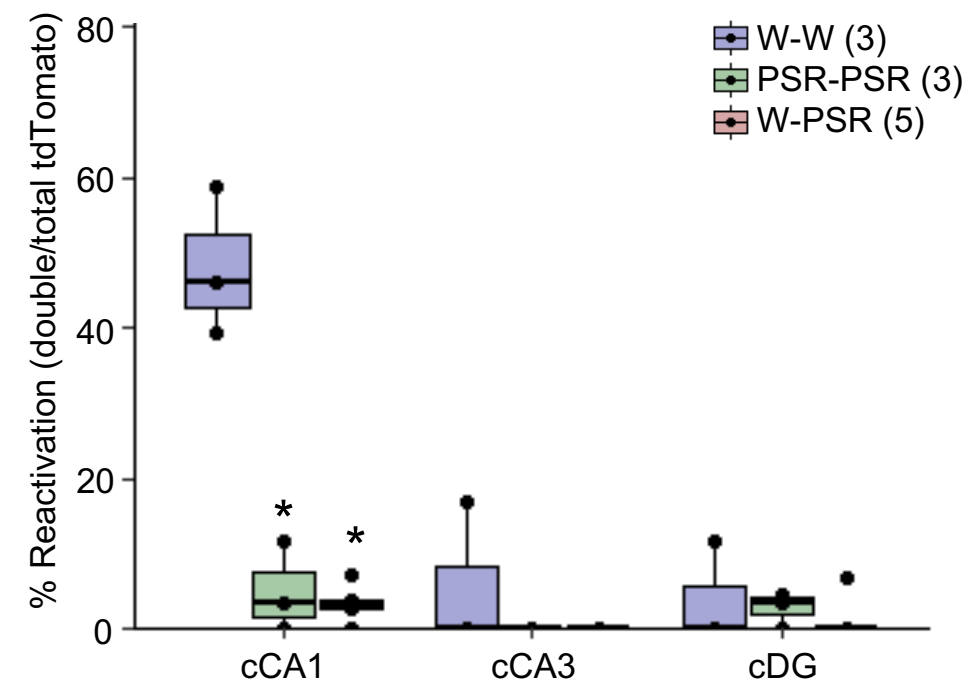

https://mc.manuscriptcentral.com/jsleep 
1 A

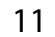

12

13
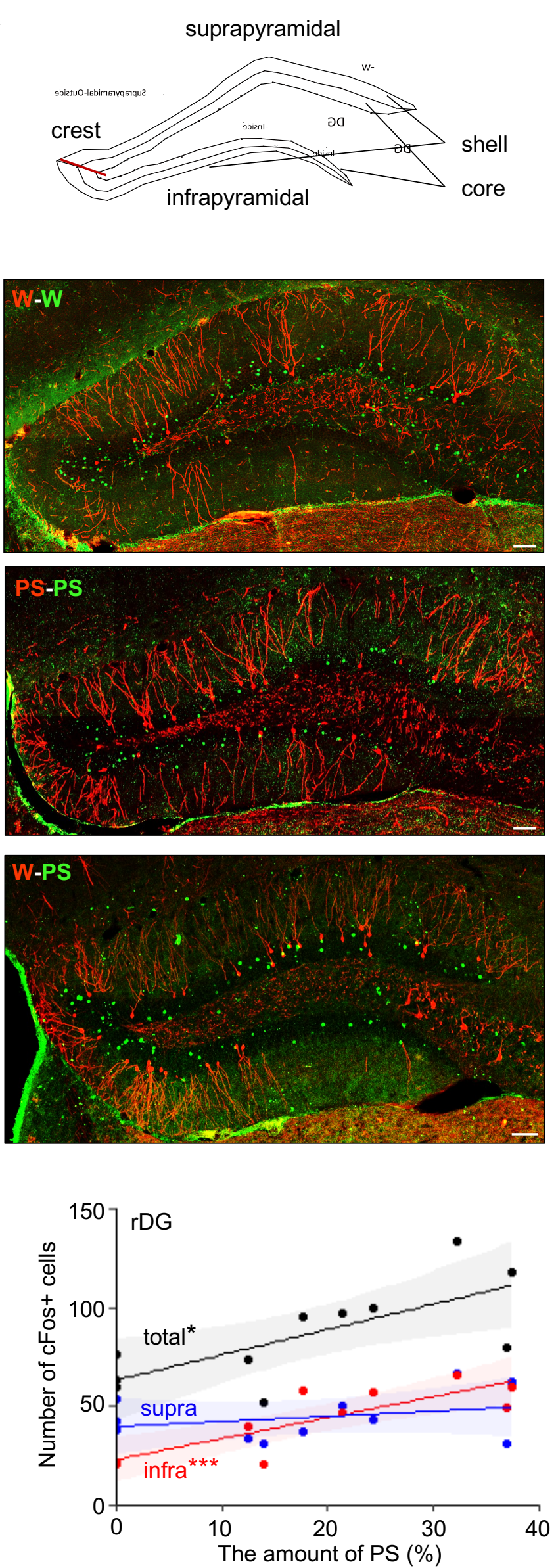

\section{C rDG}
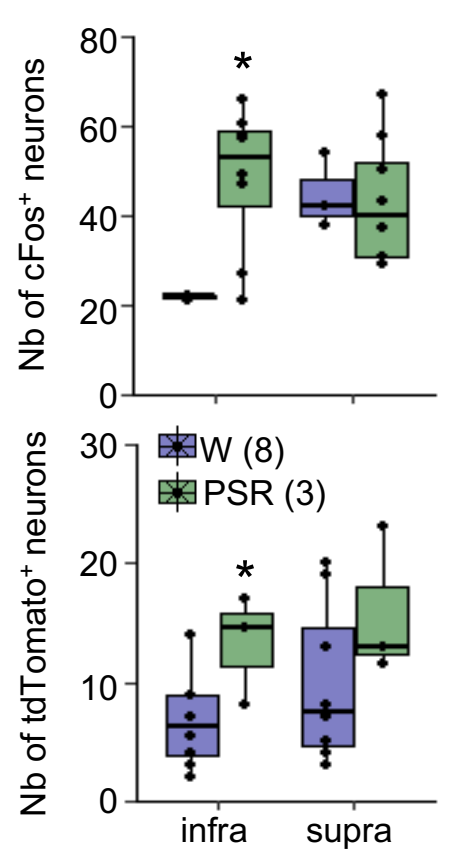

D CDG
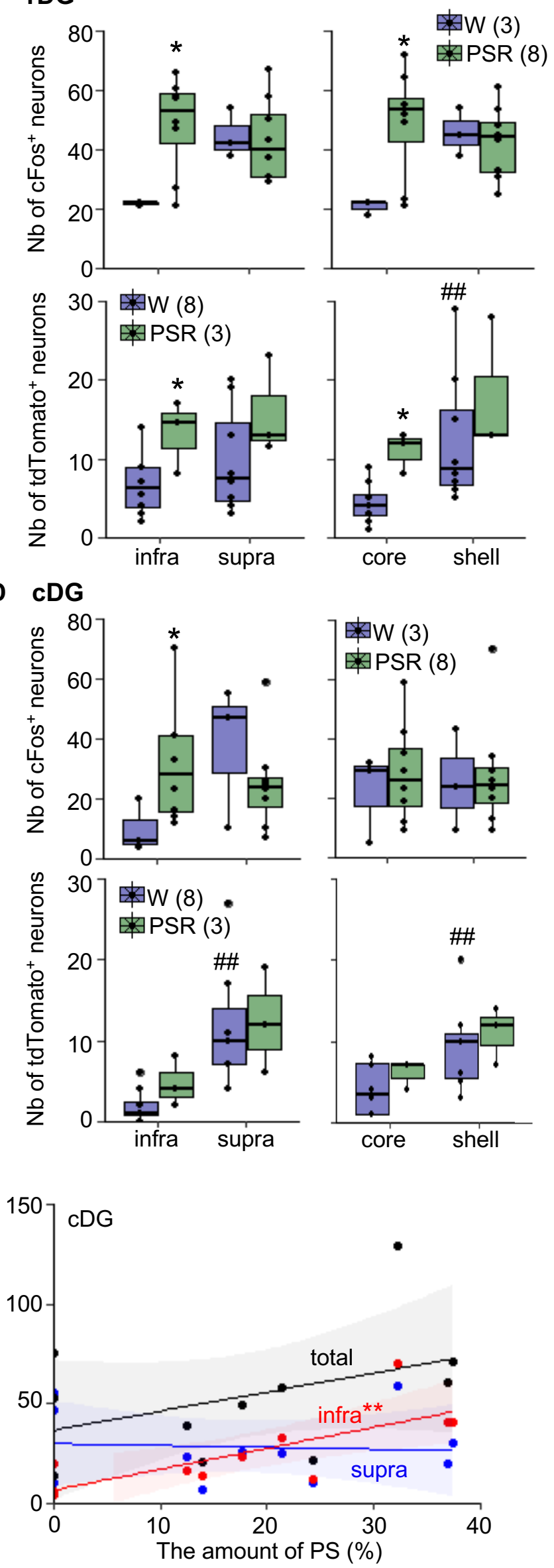